\title{
ON QUINT-CANONICAL BIRATIONALITY OF IRREGULAR THREEFOLDS
}

\author{
JHENG-JIE CHEN, JUNGKAI ALFRED CHEN, MENG CHEN, ZHI JIANG
}

\begin{abstract}
Let $X$ be a complex smooth projective threefold of general type. Assume $q(X)>0$. We show that the $m$-canonical map of $X$ is birational for all $m \geq 5$.
\end{abstract}

\section{INTRODUCTION}

In the study of birational geometry, the canonical divisor $K$, together with pluri-canonical divisor $m K$ for any $m \in \mathbb{Z}$, plays the central role. Let $V$ be a nonsingular projective variety of dimension $n$. For any integer $m$, denote by $\varphi_{m, V}$ the $m$-canonical map of $V$. The geometry of $\varphi_{m, V}$ and its variants draw a lot of attention in recent years. For varieties of general type, it is proved, independently by HaconMcKernan [HM06], Takayama [Taka] and Tsuji Tsuji, that there is a constant $\widetilde{r}_{n}$ depending only on the dimension $n(n>2)$ such that $\varphi_{m, V}$ is birational for all $m \geq \widetilde{r}_{n}$. The following question is fundamental in birational geometry:

Question 1.1. For any integer $n \geq 3$, find the optimal constant $r_{n}$ so that, for all nonsingular projective $n$-folds of general type, $\varphi_{m}$ is birational onto its image for all $m \geq r_{n}$.

It is well-known that $r_{1}=3$ and $r_{2}=5$. The results of Jungkai Chen and Meng Chen show that $r_{3} \leq 57$ (cf. [CC3, C18). It is clear that $r_{n}$ is non-decreasing with respect to $n$. However it seems to be very difficult to find explicit bounds in higher dimensions.

On the other hand, if one works on irregular varieties of general type, according to the method and the principle developed in [CH3], together with some more recent results of Jiang and Sun ([JS]), there are many interesting results about effective birationality of pluricanonical maps

The first and second authors were partially supported by National Center for Theoretical Sciences and Ministry of Science and Technology. The third author was supported by National Natural Science Foundation of China (\#11571076, \#11731004) and Program of Shanghai Subject Chief Scientist (\#16XD1400400). The forth author was supported by National Natural Science Foundation of China (\#11871155, \#11731004) and the program "Recruitment of global experts". 
depending only on the fiber dimensions of the Albanese maps. We summarize some results here:

1) if $X$ is of general type and of maxiaml Albanese dimension, then $\left|3 K_{X}\right|$ induces a birational map (see [CH4 and [JLT]);

2 ) if $X$ is of general type and a general fiber of the Albanese morphism is of dimension 1 , then $\left|4 K_{X}\right|$ induces a birational map (see [JS]).

Hence it naturally grows out the following question:

Question 1.2. Is it true that $\varphi_{m}$ is birational for all $m \geq r_{n}$ and for all irregular varieties of general type of dimension $n+1$.

Question 1.2 is known to be true when $n=1$. The purpose of this article is to prove the following result which gives a positive answer to the case of $n=2$.

Theorem 1.3. Let $X$ be a nonsingular irregular threefold of general type. Then $\varphi_{5, X}$ is birational.

Remark 1.4. This result is optimal since the 4-canonical map of any 3 -fold fibered by surfaces of general type with $K^{2}=1$ and $p_{g}=2$ is not birational. Moreover, the proof of Theorem 1.3 is essentially numerical hence it works for all the linear systems $\left|5 K_{X} \otimes P\right|$ for all $P \in \operatorname{Pic}^{0}(X)$.

Before going into details, we first summarize some more known results about threefolds of general type:

1) Chen and Hacon proved that if $X$ is an irregular threefold of general type with $\chi\left(\omega_{X}\right)>0$, then $\left|5 K_{X}\right|$ induces a biratioal map ([CH3, Theorem 1.1]);

2) By classical results on pluricanonical maps on surfaces (see Theorem 2.1 below), one sees that if $X$ is a threefold of general type and the Albanese morphism $a_{X}$ of $X$ factors through a curve of genus $\geq 2$, then $\left|5 K_{X}\right|$ induces a birational map.

Combining known results, in order to prove Theorem 1.3, it is sufficient to consider the case when $X$ satisfies the following conditions:

$(\dagger) \chi\left(\omega_{X}\right) \leq 0$ and the image of the Albanese morphism is an elliptic curve $E$.

For $X$ satisfying condition $(\dagger)$, a general fiber $F$ of $a_{X}$ is a smooth surface of general type. We denote by $F_{0}$ the minimal model of $F$.

We now briefly explain the idea of the proof of Theorem 1.3 and the organization of the article. The method based on GV-sheaves and Mregular sheaves due to Chen-Hacon and Pareschi-Popa is quite powerful to deal with pluricanonical systems of varieties with large irregularity, 
say varieties of maximal Albanese dimension or varieties of Albanese fiber dimension 1 (see [CH2], [CH3], [JLT], [JS], [PP], [BLNP]). However, the situation here is more complicated and it seems to be difficult to conclude simply by studying the positive properties of the pushforward of pluricanonical sheaves on the Albanese varieties. On the other hand, Reider type theorems work perfectly well for adjoint linear systems on surfaces (see [Laz]). Here, the idea is to combine both methods. We first show that positivity on the Albanese variety gives strong constraints on the structure of $X$ whose $\left|5 K_{X}\right|$ is difficult to study. Then we use twisted bicanonical or tri-canonical system to produce a pencil $|L|$ of $X$ and apply Tankeev's principle, we need to show that $\left|5 K_{X}\right|$ induces a birational map on a general member $S \in|L|$ to conclude that $\left|5 K_{X}\right|$ is birational. In the last step, we extensively apply a version of Reider theorem due to Langer Lan together with volume estimation of big divisors on irregular varieties, which is available due to recent progress of Severi type inequalities ([BPS], [J2], [JP]).

The structure of the article is as follows. In Section 2, we recall some elementary but useful known facts of surfaces and irregular threefolds. We start the study of twisted pluricanonical systems in Section 3. More precisely, we consider the sheaves of the form $a_{X *}\left(\mathscr{O}_{X}(M) \otimes \mathscr{I}_{x}\right)$, where $M$ is an divisor on $X$ such that $a_{X *}\left(\mathscr{O}_{X}(M)\right)$ is ample. In fact, we mainly consider $M=2 K_{X}$ or $M=3 K_{X}$ in the sequel. Suppose that either $a_{X *}\left(\mathscr{O}_{X}(2 K) \otimes \mathscr{I}_{x}\right)$ or $a_{X *}\left(\mathscr{O}_{X}(3 K) \otimes \mathscr{I}_{x}\right)$ is $M$-regular, then we prove that $\left|5 K_{X}\right|$ is birational.

If, on the other hand, $a_{X *}\left(\mathscr{O}_{X}(M) \otimes \mathscr{I}_{x}\right)$ is not $M$-regular, then we prove in Section 4 that there is a decomposition of $M=L_{M}+\mathscr{D}_{M, 0}$ into a sum of divisors such that $|M+P|=\left|L_{M}\right|+\mathscr{D}_{M, P}$ and $\mathscr{D}_{M, P}-\mathscr{D}_{M, 0}=$ $P$. Moreover, the pushforward $a_{X *}\left(\mathscr{O}_{X}(M)\right)$ has certain tensor-product structure. With these type of structures, together with the study of twisted bi-canonical map $\left|2 K_{X}+P\right|$, we are able to prove the main theorem in the case that $p_{g}(F) \neq 0$ in Section 5. Similarly, in Section 6 , we are able to prove the theorem by studying twisted tri-canonical map $\left|3 K_{X}+P\right|$ when $p_{g}(F)=0$.

1.1. Notations. Let $X$ be a smooth projective variety. We denote by $a_{X}: X \rightarrow A_{X}$ the Albanese variety of $X$ and $\operatorname{Pic}^{0}(X)$ the Picard variety of $X$. We denote by $\sim$ linear equivalence of divisors and $\equiv$ numerical equivalence of divisors.

Acknowledgements. The forth author would like to thank National Center for Theoretical Sciences in Taipei for the warm hospitality in January 2019. 


\section{Preliminary Results}

2.1. Some well-known results on surfaces. We recall a classical theorem due to Bombieri, Miyaoka and many others (see for instance [BPV, Section 7]).

Theorem 2.1. Let $F_{0}$ be a minimal surface of general type. If $k \geq 5$, then $\varphi_{k, F_{0}}$ is a birational morphism onto its canonical model. If $k \geq 3$, $\varphi_{k, F_{0}}$ is birational, except in the following cases:

1) $\left(K_{F_{0}}^{2}, P_{g}\left(F_{0}\right)\right)=(2,3)$, then $\varphi_{3}$ is a morphism of degree 2 and the canonical model of $F_{0}$ is a hypersurface of degree 8 in $\mathbb{P}(1,1,1,4)$;

2) $\left(K_{F_{0}}^{2}, P_{g}\left(F_{0}\right)\right)=(1,2)$, then $\varphi_{3}$ and $\varphi_{4}$ are maps of degree 2 and the canonical model of $F_{0}$ is a hypersurface of degree 10 in $\mathbb{P}(1,1,2,5)$;

Proposition 2.2. 1) If $p_{g}(F) \neq 0$, then the linear system $\left|2 K_{F_{0}}\right|$ is base point free ([BPV, Theorem 7.4]).

2) If $K_{F_{0}}^{2} \geq 2$, then $\left|3 K_{F_{0}}\right|$ is base point free ([BPV, Theorem 5.1]).

In this article, we will frequently apply Reider type results of birationality criterion for adjoint line bundles on surfaces. Let $S$ be a smooth projective surface and $D$ a nef $\mathbb{Q}$-divisor. Let $x_{1}$ and $x_{2}$ be two different closed points of $S$. The following theorem (see [Mas] or Lan, Theorem 0.1]) gives an effective condition for point separation of $\left|K_{S}+\lceil D\rceil\right|$.

Theorem 2.3. Assume that $D^{2}>8$. If $\left|K_{S}+\lceil D\rceil\right|$ does not separate $x_{1}$ and $x_{2}$, then there exists a curve $C$ passing through $x_{1}$ and $x_{2}$ such that

$$
(D \cdot C) \leq \frac{4}{1+\sqrt{1-\frac{8}{D^{2}}}} .
$$

In applying Theorem 2.3 in our proof, a crucial step is to estimate the lower bound of volumes of nef $\mathbb{Q}$-divisors on an irregular threefold. The following useful results are special forms of the main result in [J2, Remark 3.3 and Corollary 3.4].

Proposition 2.4. Let $f: X \rightarrow A$ be a fibration from a smooth projective $n$-fold to an abelian variety. Assume that $D$ is a nef and big divisor on $X$.

(1) If $\left.D\right|_{X_{s}}$ is base point free where $X_{s}$ is a general fiber of $f$ over its image and that $f_{*} \mathscr{O}_{X}(D)$ is a semi-stable ample vector bundle of rank $r$. Then $D^{n} \geq \frac{n}{r}\left(\left.D\right|_{X_{s}}\right)^{n-1} h^{0}(X, D)$. 
(2) If the connected component of a general fiber of $f$ over its image is a curve of genus $g \geq 2$, then $\operatorname{vol}(D) \geq 2(n-1) ! h^{0}\left(A, f_{*} D \otimes\right.$ $Q)$, where $Q \in \operatorname{Pic}^{0}(A)$ general.

Lemma 2.5. Let $F_{0}$ be a minimal surface of general type such that the linear system $\left|m K_{F_{0}}\right|$ contains an irreducible and smooth curve for some $m \geq 2$ and $\left|(m+1) K_{F_{0}}\right|$ induces a birational map. Then a general curve $C \in\left|m K_{F_{0}}\right|$ is not hyperelliptic.

Proof. Let $C \in\left|m K_{F_{0}}\right|$ be a general member, then $\left|(m+1) K_{F_{0}}\right|_{\mid C}$ induces a birational map of $C$. On the other hand, considering

$$
0 \rightarrow K_{F_{0}} \rightarrow K_{F_{0}}+C \rightarrow K_{C} \rightarrow 0
$$

we conclude that $\left|K_{F_{0}}+C\right|_{C}=\left|(m+1) K_{F_{0}}\right|_{C}$ is a sublinear system of $\left|K_{C}\right|$. Hence $C$ is not hyperelliptic.

Remark 2.6. By [BPV, Section 7], we know that if $p_{g}\left(F_{0}\right)>0$, then $\left|2 K_{F_{0}}\right|$ is base point free and $\left|3 K_{F_{0}}\right|$ induces a birational map of $F_{0}$ except $K_{F_{0}}^{2}=1$ and $p_{g}\left(F_{0}\right)=2$ or $K_{F_{0}}^{2}=2$ and $p_{g}\left(F_{0}\right)=3$. If $p_{g}\left(F_{0}\right)=0$, then $\left|4 K_{F_{0}}\right|$ is always birational and $\left|3 K_{F_{0}}\right|$ is always base point free when $K_{F_{0}}^{2} \geq 2$. If $p_{g}\left(F_{0}\right)=0$ and $K_{F_{0}}^{2}=1$, then $\left|3 K_{F_{0}}\right|$ has no fixed component and a general member is irreducible and smooth (see [Cat]).

2.2. Plurigenera of Irregular Threefolds. Some cases of the following lemma (see for instance [CC1, 4.4]) are known.

Lemma 2.7. Let $X$ be a smooth irregular threefold of general type. Then we have $P_{k+1}(X)>P_{k}(X) \geq 1$ for all $k \geq 2$.

Proof. If $X$ is of Albanese fiber dimension $\leq 1$, then by [CH2] and [JS], we know that $V^{0}\left(\omega_{X}, a_{X}\right)=\left\{P \in \operatorname{Pic}^{0}(X) \mid h^{0}\left(\omega_{X} \otimes P\right) \neq 0\right\}$ has an irreducible component $T$ of dimension $\geq 1$. Considering the map

$$
\bigcup_{P \in T} H^{0}\left(X, \omega_{X} \otimes P\right) \otimes H^{0}\left(X, \omega_{X}^{k} \otimes P^{-1}\right) \rightarrow H^{0}\left(X, \omega_{X}^{k+1}\right),
$$

we conclude that $P_{k+1}(X)>P_{k}(X) \geq 1$ for any $k \geq 2$.

We now consider the case when the image of $a_{X}$ is a curve, where we take the Stein factorization

$$
a_{X}: X \stackrel{f}{\rightarrow} C \stackrel{g}{\rightarrow} A_{X},
$$

and let $F$ be a general fiber of $f$.

First recall that by [CH1, Lemma 4.1], $g_{*} f_{*} \omega_{X}^{k}$ and $g_{*} f_{*}\left(\omega_{X}^{k} \otimes Q\right)$ are non-trivial $\mathrm{IT}^{0}$ sheaves on $A_{X}$ for all $k \geq 2$ and for all $Q \in \widehat{A}_{X}$. 1

\footnotetext{
${ }^{1}$ Chen and Hacon proved this when $C$ is an elliptic curve but their argument works in general.
} 
If $p_{g}(F) \neq 0$, then $a_{X *} \omega_{X}$ is a non-trivial GV-sheaf. Hence there exists $Q \in \widehat{A}_{X}$ such that $H^{0}\left(X, K_{X}+Q\right) \neq 0$. A non-zero section $s \in H^{0}\left(X, K_{X}+Q\right)$ induces a short exact sequence:

$$
0 \rightarrow f_{*} \omega_{X}^{k} \stackrel{\cdot s}{\rightarrow} f_{*}\left(\omega_{X}^{k+1} \otimes Q\right) \rightarrow \mathscr{Q}_{k} \rightarrow 0 .
$$

We notice that $P_{k+1}(F)>P_{k}(F)$ for all $k \geq 2$, hence $\mathscr{Q}_{k}$ is a non-trivial IT $^{0}$ coherent sheaf on $C$. Hence

$$
\begin{aligned}
P_{k+1}(X) & =h^{0}\left(X,(k+1) K_{X}+Q\right)=h^{0}\left(A_{X}, g_{*} f_{*}\left(\omega_{X}^{k+1} \otimes Q\right)\right) \\
& =h^{0}\left(A_{X}, g_{*} f_{*} \omega_{X}^{k}\right)+h^{0}\left(A_{X}, \mathscr{Q}_{k}\right) \\
& >h^{0}\left(A_{X}, g_{*} f_{*} \omega_{X}^{k}\right)=P_{k}(X) .
\end{aligned}
$$

If $p_{g}(F)=0$, then $q(F)=0$ and hence $f_{*} \omega_{X}=R^{1} f_{*} \omega_{X}=0$. We have

$$
\begin{aligned}
\chi\left(X, \omega_{X}\right) & =\operatorname{deg}\left(f_{*} \omega_{X}\right)-\operatorname{deg}\left(R^{1} f_{*} \omega_{X}\right)+\operatorname{deg}\left(R^{2} f_{*} \omega_{X}\right) \\
& =\operatorname{deg} \omega_{C} \geq 0 .
\end{aligned}
$$

We denote by $Y$ the minimal model of $X$. Then $\chi\left(Y, \mathscr{O}_{Y}\right)=\chi\left(X, \mathscr{O}_{X}\right) \leq$ 0 . Since $K_{Y}^{3}>0$, by Reid's Riemann-Roch formula, $P_{2}(X)=\frac{1}{2} K_{Y}^{3}-$ $3 \chi\left(Y, \mathscr{O}_{Y}\right)+\mathscr{R}_{2}>0$ and for all $k \geq 2$,

$$
P_{k+1}(X)-P_{k}(X)=\frac{k^{2}}{2} \cdot K_{Y}^{3}-2 \chi\left(Y, \mathscr{O}_{Y}\right)+\mathscr{R}_{k+1}
$$

where $\mathscr{R}_{2}, \mathscr{R}_{k+1}$ denotes the contribution of singularities which are always non-negative. It follows immediately that $P_{k+1}(X)>P_{k}(X) \geq 1$ for all $k \geq 2$.

\section{Twisted Pluricanonical systems}

Proposition 3.1. Let $X$ be a smooth projective threefold of general type satisfying Condition $(\dagger)$. If there exists an open dense subset $U$ of $X$ such that, for each point $x \in U, E_{3, x}=a_{*}\left(\omega_{X}^{3} \otimes \mathscr{I}_{x}\right)$ is M-regular, then $\varphi_{5, X}$ is birational.

Proof. For any $x \in U, E_{3, x}$ is M-regular and hence is continuously globally generated (see [PP, Proposition 2.13]). Then, by [CH3, Corollary 2.4], there exists an open dense subset $V$ of $U$ such that the linear system $\left|5 K_{X}\right|$ separates any two points $x, y$ of $V$ in different fibers of $a_{X}$. Moreover, if $x, y \in V$ are two different points in a general fiber $F$ of $a_{X}$ and if $\left|3 K_{F}\right|$ separates $x$ and $y$, then $\left|5 K_{X}\right|$ also separates $x$ and $y$.

By Theorem 2.1, $\varphi_{3, F}$ is birational unless $F$ is either a $(1,2)$-surface or a $(2,3)$-surface. Moreover, for these two classes of surfaces, $\left|3 K_{F}\right|$ induces a map of degree 2 . 
Assume, to the contrary, that $\varphi_{5, X}$ is non-birational. We want to deduce a contradiction.

Then the linear system $\left|5 K_{X}\right|$ induces a map $\varphi_{5}: X \rightarrow Y$ of degree 2. We are going to argue by contradiction to show that it is absurd.

Note that $\varphi_{5, X}: X \rightarrow \hat{Y}$ must be generically finite of degree 2 . Hence there is a birational involution $\tau$ on $X$ switching two fibers of $\varphi_{5}$. Modulo birational modifications, we may assume that $\tau$ is a biregular involution and the quotient $Y=X / \tau$ is also a smooth projective variety. The involution $\tau$ preserve general fibers of the Albanese morphism $a_{X}$ and hence we have a commutative diagram:

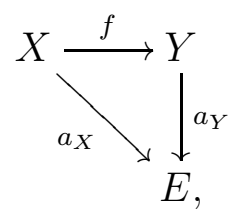

where $f$ is the quotient morphism of $\tau$. By assumption, $\varphi_{5, X}$ factors through $f$. There exists a line bundle $\mathscr{L}$ on $Y$ such that $\omega_{X}=f^{*}\left(\omega_{Y} \otimes\right.$ $\mathscr{L}$ ) and $f_{*} \mathscr{O}_{X}=\mathscr{O}_{Y} \oplus \mathscr{L}^{-1}$. Thus we have

$$
f_{*} \omega_{X}^{5}=\left(\omega_{Y}^{\otimes 5} \otimes \mathscr{L}^{\otimes 5}\right) \oplus\left(\omega_{Y}^{\otimes 5} \otimes \mathscr{L}^{\otimes 4}\right)
$$

where $\omega_{Y}^{\otimes 5} \otimes \mathscr{L}^{\otimes 5}$ (resp. $\omega_{Y}^{\otimes 5} \otimes \mathscr{L}^{\otimes 4}$ ) correspond to $\tau$-invariant (resp. $\tau$-anti-invariant) sections of $\omega_{X}^{5}$.

Since $\left|5 K_{F}\right|$ induces a birational map of $F$ and $a_{X *} \omega_{X}^{\otimes 5}$ is ample, both $a_{Y *}\left(\omega_{Y}^{\otimes 5} \otimes \mathscr{L}^{\otimes 5}\right)$ and $a_{Y *}\left(\omega_{Y}^{\otimes 5} \otimes \mathscr{L}^{\otimes 4}\right)$ are ample. Since both the ample sheaves on $E$ have global sections, they are lift to global ones of $\omega_{X}^{\otimes 5}$. Hence direct summand of $a_{X *} \omega_{X}^{\oplus 5}$, it is ample. Hence $H^{0}\left(X, 5 K_{X}\right)$ contains a section separating the two points on the general fiber of $f$, which means however that $\varphi_{5, X}$ does not factor birationally through $f$, a contradiction.

Hence, in order to prove the main theorem, we will mainly work under the condition that $E_{3, x}$ is not M-regular in the rest of the article. But some variant of Proposition 3.1 is also needed to deal with some special cases.

Proposition 3.2. Let $X$ be a smooth projective threefold of general type satisfying Condition ( $\dagger)$. Let $F$ be a general fiber of $a_{X}$. Assume that, for general point $x \in X, a_{X *}\left(\omega_{X}^{2} \otimes \mathscr{I}_{x}\right)$ is M-regular. Then $\left|5 K_{X}\right|$ induces a birational map. 
Proof. Since $a_{X *}\left(\omega_{X}^{2} \otimes \mathscr{I}_{x}\right)$ is M-regular for general $x \in X$, the evaluation map

$$
\bigoplus_{P \in W} H^{0}\left(X, a_{X *}\left(\omega_{X}^{2} \otimes \mathscr{I}_{x}\right) \otimes P\right) \otimes P^{-1} \rightarrow a_{X *}\left(\omega_{X}^{2} \otimes \mathscr{I}_{x}\right)
$$

is surjective for any open subset $W$ of $\operatorname{Pic}^{0}(X)$. Since $p_{2}(F) \neq 0$ for general fiber $F$ of $a_{X}$ and $\operatorname{Bs}\left|2 K_{F}\right|$ is a proper closed subset of $F$, for any two general points $x, y$ of $X$ lying in different general fibers of $a_{X}$, there exists a point $P \in W$ and a divisor $D_{P} \in\left|2 K_{X}+P\right|$ passing through $x$ and not passing through $y$. As $y \in X$ is general, there exists a smaller open subset $W^{\prime} \subset W$ such that $y$ is not contained in the base locus of $\left|3 K_{X}-Q\right|$ for any $Q \in W^{\prime}$. Thus, by considering the natural map $\left|2 K_{X}+P\right| \times\left|3 K_{X}-P\right| \rightarrow\left|5 K_{X}\right|$, there exists a section in $H^{0}\left(5 K_{X}\right)$ separating $x$ and $y$.

From now on, we always assume that $x$ and $y$ are two general points in a general fiber $F$ of $a_{X}$.

If $\left|2 K_{F}\right|$ can separate $x$ and $y$, then $\left|5 K_{X}\right|$ can separate $x$ and $y$ by the argument of [CH3, Corollary 2.4]. Assume that $\left|3 K_{F}\right|$ induces a birational map. Take $x, y \in F$ to be two general points in a general fiber $F$ and that $x$ and $y$ can not be separated by $\left|2 K_{F}\right|$. Since $a_{X *} \omega_{X}^{3}$ is $\mathrm{IT}^{0}$, it is M-regular and, by taking the localization, the natural map

$$
\bigoplus_{P \in W} H^{0}\left(X, 3 K_{X}+P\right) \rightarrow H^{0}\left(F, 3 K_{F}\right)
$$

is surjective for any open subset $W$ of $\operatorname{Pic}^{0}(X)$. Find a section $f \in$ $H^{0}\left(F, 3 K_{F}\right)$ such that $f(x)=0$ and $f(y) \neq 0$. We then choose $\widetilde{f}_{i} \in$ $H^{0}\left(X, 3 K_{X}+P_{i}\right), 1 \leq i \leq k$, such that $\left.\sum_{i} \widetilde{f}_{i}\right|_{F}=f$. Since $a_{X *}\left(\omega_{X}^{2} \otimes \mathscr{I}_{x}\right)$ is M-regular, $V^{1}\left(a_{X *}\left(\omega_{X}^{2} \otimes \mathscr{I}_{x}\right)\right)=\emptyset$. For a general $x \in X$, the short exact sequence

$$
0 \rightarrow a_{X *}\left(\omega_{X}^{2} \otimes \mathscr{I}_{x}\right) \otimes P \rightarrow a_{X *} \omega_{X}^{2} \otimes P \rightarrow \mathbb{C}_{x} \rightarrow 0
$$

implies the surjective map $H^{0}\left(2 K_{X}+P\right) \rightarrow \mathbb{C}_{x}$ for any $P \in \operatorname{Pic}^{0}(X)$, which means that, as a general point of $X, x$ is not in the base locus of $\left|2 K_{X}+P\right|$ for any $P \in \operatorname{Pic}^{0}(X)$. We then take $\widetilde{g}_{i} \in H^{0}\left(X, 2 K_{X}-P_{i}\right)$, $1 \leq i \leq k$, such that $\widetilde{g}_{i}(y)=1$ for $1 \leq i \leq k$. As $x$ and $y$ can not be separated by $\left|2 K_{F}\right|$, we see that $\widetilde{g}_{i}(x) \neq 0$ for $1 \leq i \leq k$. We then conclude that $h:=\sum_{1 \leq i \leq k} \widetilde{g}_{i} \widetilde{f}_{i} \in H^{0}\left(X, 5 K_{X}\right)$ satisfies the property that $h(x)=0$ and $h(y) \neq 0$.

When $\left|3 K_{F}\right|$ does not induce a birational map for $F$, the argument of the second part in the proof of Proposition 3.1 works as well. So we have completed the proof. 


\section{ABEL-JACOBI MAP INDUCED BY FiXeD DIVISORS}

In this section, we use Abel-Jacobi map to analyze the fixed divisors of twisted pluricanonical systems of an irregular variety $X$ with $q(X)=$ 1. Lemma 4.4 and 4.9 will be frequently used in next sections.

4.1. Setting. Let $X$ be a smooth projective variety of general type and assume that the Albanese morphism $a_{X}: X \rightarrow E$ of $X$ is a fibration onto an elliptic curve. Denote by $F$ a general fiber of $a_{X}$.

Denote by $\mathscr{P}$ the Poincaré bundle, on $X \times \widehat{E}$, which is pulled back from $E \times \widehat{E}$. Assume that we have a divisor $M$ on $X$ such that $H^{0}(X, M \otimes P) \neq 0$ for general $P \in \widehat{E}$. Then $p_{2 *}\left(p_{1}^{*} M \otimes \mathscr{P}\right)$ is a non-trivial vector bundle on $E$, where $p_{1}$ and $p_{2}$ are the natural projections from $X \times \widehat{E}$ to $X$ and $\widehat{E}$ respectively. We have the relative evaluation map:

$$
p_{2}^{*} p_{2 *}\left(p_{1}^{*} M \otimes \mathscr{P}\right) \stackrel{v}{\rightarrow} p_{1}^{*} M \otimes \mathscr{P} .
$$

Then there exists an ideal sheaf $\mathscr{I}_{M}$ on $X \times \widehat{E}$ so that $\mathscr{I}_{M} \otimes p_{1}^{*} M \otimes \mathscr{P}$ is the image of $v$. Let $\mathscr{Z}_{M}$ be the scheme of $X \times \widehat{E}$ defined by $\mathscr{I}_{M}$.

The following lemma is a crucial observation. Note that a similar observation plays a crucial role in BLNP] as well, though the context is completely different.

Lemma 4.2. Keep the setting as in 4.1. Assume that $a_{X *} \mathscr{O}_{X}(M)$ is an ample vector bundle on $E$ and $\mathscr{Z}_{M}$ does not dominate $X$. Then there exists an open dense subset $U$ of $X$ such that for any $x \in U$, $a_{X *}\left(\mathscr{O}_{X}(M) \otimes \mathscr{I}_{x}\right)$ is M-regular. In particular, $a_{X *}\left(\mathscr{O}_{X}(M) \otimes \mathscr{I}_{x}\right)$ is ample on $E$.

Proof. For a general $P \in \widehat{E}$, one has

$$
p_{2 *} \mathscr{O}_{X}\left(p_{1}^{*} M \otimes \mathscr{P}\right) \otimes \underline{\mathbb{C}}_{P} \simeq H^{0}(X, M \otimes P) .
$$

Thus, if $\mathscr{Z}_{M}$ does not dominate $X$, the following base locus

$$
\mathscr{B}:=\{(x, P) \in X \times \widehat{E}|x \in \operatorname{Bs}| M+P \mid\}
$$

does not dominate $X$ and denote by $U$ the open dense subset $X-p_{1}(\mathscr{B})$. Then, for any $x \in U$, we have the short exact sequence

$$
0 \rightarrow a_{X *}\left(\mathscr{O}_{X}(M) \otimes \mathscr{I}_{x}\right) \rightarrow a_{X *} \mathscr{O}_{X}(M) \rightarrow \underline{\mathbb{C}}_{a_{X}(x)} \rightarrow 0 .
$$

By definition of $\mathscr{B}$, we conclude that $H^{0}\left(X, a_{X *} \mathscr{O}_{X}(M) \otimes P\right) \rightarrow \mathbb{C}_{a_{X}(x)}$ is surjective for any $P \in \widehat{E}$. By assumption, $a_{X *} \mathscr{O}_{X}(M)$ is an ample vector bundle on $E$. By considering the long exact sequence, we conclude that $H^{1}\left(E, a_{X *}\left(\mathscr{O}_{X}(M) \otimes \mathscr{I}_{x}\right) \otimes P\right)=0$ for any $P$. Thus $a_{X *}\left(\mathscr{O}_{X}(M) \otimes \mathscr{I}_{x}\right)$ is M-regular and, in particular, ample on $E$. 
Now the rest of this section is devoted to studying in detail the case when $\mathscr{Z}_{M}$ dominates $X$ via the first projection. We denote by $\mathscr{D}_{M}$ the union of irreducible components, of $\mathscr{Z}_{M}$, each of which dominates $X$. We observe that each irreducible component of $\mathscr{D}_{M}$ is a divisor of $X \times \widehat{E}$ dominating both $X$ and $E$.

Lemma 4.3. Keep the setting as in 4.1. Assume that $\mathscr{Z}_{M}$ dominates $X$. Then $\mathscr{D}_{M}$ is reduced and irreducible and, for $P \in \widehat{E}$ general, $\mathscr{D}_{M, P}:=\left.\mathscr{D}_{M}\right|_{X \times\{P\}}$ is also reduced and irreducible. Moreover, the Abel-Jacobi map induced by $\mathscr{D}$

$$
\begin{aligned}
\Psi: & \widehat{E} \longrightarrow \widehat{E} \\
& P \rightarrow \mathscr{O}_{X}\left(\mathscr{D}_{M, P}-\mathscr{D}_{M, 0}\right)
\end{aligned}
$$

is the identity and hence $\mathscr{D}_{M, P}=\mathscr{D}_{M, 0}+P$. Fix a general $P$, we set $L_{M}:=M+P-\mathscr{D}_{M, P}$. Then $|M+Q|=\left|L_{M}\right|+\mathscr{D}_{M, Q}$ for general $Q$. Furthermore, $V^{0}\left(L_{M}\right) \neq \widehat{E}$.

Proof. First we prove that each irreducible component of $\mathscr{D}_{M}$ is reduced. Otherwise let $k \mathscr{D}_{1}$ (with $k \geq 2$ ) be an irreducible component of $\mathscr{D}_{M}$. Fix a general element $P \in \widehat{E}$, then $k \mathscr{D}_{1 P}$ is a fixed divisor on $X$. We notice that $\mathscr{D}_{1}$ is a family of divisors of $X$ parametrized by $\widehat{E}$ and the Abel-Jacobi maps:

$$
\begin{aligned}
\Phi: \widehat{E} & \longrightarrow \widehat{E} \\
P & \rightarrow \mathscr{O}_{X}\left(\mathscr{D}_{1 P}-\mathscr{D}_{10}\right)
\end{aligned}
$$

is a non-trivial isogeny of $\widehat{E}$. Hence we may take two general elements $Q_{1}$ and $Q_{2}$, different from $P$, such that $\Phi\left(Q_{1}\right)+\Phi\left(Q_{2}\right)=2 \Phi(P)$. Hence $\mathscr{D}_{1 Q_{1}}+\mathscr{D}_{1 Q_{2}}$ is rational equivalent to $2 \mathscr{D}_{1 P}$. This contradicts the fact that $k \mathscr{D}_{1 P}$ is a fixed divisor.

If $\mathscr{D}_{M}$ has two different irreducible components $\mathscr{D}_{1}$ and $\mathscr{D}_{2}$. We still consider the Abel-Jacobi maps

$$
\begin{aligned}
\Phi_{i}: & \widehat{E} \longrightarrow \widehat{E} \\
& P \rightarrow \mathscr{O}_{X}\left(\mathscr{D}_{i P}-\mathscr{D}_{i 0}\right),
\end{aligned}
$$

for $i=1,2$. Both $\Phi_{1}$ and $\Phi_{2}$ are isogenys of $\widehat{E}$. Fix a general $P \in \widehat{E}$, then $\mathscr{D}_{1 P}+\mathscr{D}_{2 P}$ is a fixed divisor. Choose a general $Q \in \widehat{E}$ such that for some $P_{1} \in \Phi_{1}^{-1}\left(\Phi_{1}(P)-Q\right)$ and $P_{2} \in \Phi_{2}^{-1}\left(\Phi_{2}(P)+Q\right)$, the support of $\mathscr{D}_{1 P_{1}}+\mathscr{D}_{2 P_{2}}$ is different from the support of $\mathscr{D}_{1 P}+\mathscr{D}_{2 P}$. However $\mathscr{D}_{1 P}+\mathscr{D}_{2 P} \sim_{\text {rat }} \mathscr{D}_{1 P_{1}}+\mathscr{D}_{2 P_{2}}$, which is a contradiction as well.

Take the normalization $\varepsilon: \mathscr{D}^{\prime} \rightarrow \mathscr{D}_{M}$. Let $\mathscr{D}^{\prime} \stackrel{g}{\rightarrow} C \stackrel{t}{\rightarrow} \widehat{E}$ be the Stein factorization of $\mathscr{D}^{\prime} \rightarrow \widehat{E}$. If $\mathscr{D}_{M, P}$ is not irreducible for the general 
$P \in \widehat{E}$, then $\operatorname{deg} t>1$. Then we consider another Abel-Jacobi map:

$$
\begin{aligned}
\Phi_{C}: & C \longrightarrow \widehat{E} \\
& P \rightarrow \mathscr{O}_{X}\left(\varepsilon\left(\mathscr{D}_{c}^{\prime}\right)-\varepsilon\left(\mathscr{D}_{c_{0}}^{\prime}\right)\right),
\end{aligned}
$$

for some fixed point $c_{0} \in C$. Clearly $\Phi_{C}$ is dominant as well.

For the general $P \in \widehat{E}$, take two different points $c_{1}, c_{2} \in t^{-1}(P)$. We have $\varepsilon\left(\mathscr{D}_{c_{1}}^{\prime}\right)+\varepsilon\left(\mathscr{D}_{c_{2}}^{\prime}\right)$ is a sub-divisor of $\mathscr{D}_{P}$ and hence a fixed divisor. However, since $\Phi_{C}$ is dominant, there are different points $c_{3}, c_{4} \in C$ such that $\Phi_{C}\left(c_{1}\right)+\Phi_{C}\left(c_{2}\right)=\Phi_{C}\left(c_{3}\right)+\Phi_{C}\left(c_{4}\right)$ and then $\varepsilon\left(\mathscr{D}_{c_{1}}^{\prime}\right)+\varepsilon\left(\mathscr{D}_{c_{2}}^{\prime}\right) \sim_{\text {rat }} \varepsilon\left(\mathscr{D}_{c_{3}}^{\prime}\right)+\varepsilon\left(\mathscr{D}_{c_{4}}^{\prime}\right)$, a contradiction. Hence $\mathscr{D}_{M, P}$ is irreducible and reduced for the general $P \in \widehat{E}$.

The above argument actually shows that $\Psi$ is injective and hence a group isomorphism from $\widehat{E}$ to $\widehat{E}$. We prove the last statement by the same idea. Assume that $\Psi$ is different from the identity, then $\Psi$ is induced by a complex multiplication of $\widehat{E}$ and $\Psi-I d$ is also a nontrivial group isomorphism of $\widehat{E}$. We take a general $P_{0} \in \widehat{E}$ such that $h^{0}\left(X, M \otimes P_{0}\right)$ is minimal. We denote by $L_{M}$ the divisor $M+P-\mathscr{D}_{M, P_{0}}$. Then, for a general $Q \in \widehat{E}$, there exists $Q^{\prime} \in \widehat{E}$ different from $Q$, such that $\Psi\left(Q^{\prime}\right)=\Psi\left(P_{0}\right)+Q-P_{0}$. This implies that $\mathscr{D}_{Q^{\prime}}$ linear equivalent to $\mathscr{D}_{P_{0}}+Q-P_{0}$. Then $|M+Q| \supset\left|L_{M}\right|+\mathscr{D}_{Q^{\prime}}$. Since $Q \in \widehat{E}$ general, we conclude that $|M+Q|=\left|L_{M}\right|+\mathscr{D}_{Q^{\prime}}$. On the other hand, we know that $\mathscr{D}_{Q} \neq \mathscr{D}_{Q^{\prime}}$ is a fixed divisor of the linear system $|M+Q|$, thus $L_{M}-D_{Q}$ is effective, which is absurd.

We continue the study under the assumption of Lemma 4.3. Notice that, whenever we take $M=k K_{X}+a_{X}^{*} \hat{L}$ with $k \geq 2, P_{k}(F) \neq 0$ and $\hat{L}$ a nef line bundle on $E, a_{X *} \mathscr{O}_{X}(M)$ is ample (see for instance CH1, Lemma 2.1]). Then $\left.h^{0}(X, M+P)\right)=r$ is constant for all $P \in \widehat{E}$ and hence $|M+P|=\left|L_{M}\right|+\mathscr{D}_{M, P}$ for all $P \in \widehat{E}$.

We now consider the following vector bundles on $E$ :

$$
\mathscr{V}_{M}^{0}:=a_{X *} \mathscr{O}_{X}\left(L_{M}\right) \text { and } \mathscr{V}_{M}^{+}=a_{X *} \mathscr{O}_{X}\left(\mathscr{D}_{M, 0}\right)
$$

We know that a vector bundle on an elliptic curve is a direct sum of semi-stable vector bundles. Since $V^{0}\left(L_{M}\right) \neq \widehat{E}$ and $h^{0}\left(X, L_{M}\right)=r$, $\mathscr{V}_{M}^{0}$ is a direct sum of semi-stable vector bundles of negative slopes and zero slope. In particular, there is an injection

$$
\mathscr{E}_{M}^{0}:=\mathscr{O}_{E}^{\oplus r} \hookrightarrow \mathscr{V}_{M}^{0}
$$

which is induced by global sections of $L_{M}$.

Similarly, $\mathscr{V}_{M}^{+}$is a direct sum of semistable vector bundle. Since

$$
h^{0}\left(E, \mathscr{V}_{M}^{+} \otimes P\right)=h^{0}\left(X, \mathscr{D}_{M, 0} \otimes P\right)=h^{0}\left(X, \mathscr{D}_{M, P}\right)=1
$$


for any general $P \in \widehat{E}$, there exist one (and only one) ample direct summand $\mathscr{E}_{M}^{+}$of $\mathscr{V}_{M}^{+}$of degree 1 .

We have the natural map $a_{*} \mathscr{O}_{X}\left(L_{M}\right) \otimes a_{*} \mathscr{O}_{X}\left(\mathscr{D}_{M, 0}\right) \rightarrow a_{X *} \mathscr{O}_{X}(M)$ induced by multiplication of sections. Hence we have the multiplication map

$$
m:\left(\mathscr{O}_{E}^{\oplus r}\right) \otimes \mathscr{E}_{M}^{+} \hookrightarrow \mathscr{V}_{M}^{0} \otimes \mathscr{V}_{M}^{+} \rightarrow a_{X *} \mathscr{O}_{X}(M) .
$$

Recall that $|M+P|=\left|L_{M}\right|+\mathscr{D}_{M, P}$. Therefore $m$ induces an injective and hence bijective map between sections

$$
H^{0}\left(E,\left(\mathscr{O}_{E}^{\oplus r}\right) \otimes \mathscr{E}_{M}^{+} \otimes P\right) \stackrel{m}{\rightarrow} H^{0}\left(E, a_{X *} \mathscr{O}_{X}(M) \otimes P\right),
$$

for any $P \in \widehat{E}$. Notice also that both vector bundles are ample and hence have vanishing $H^{1}$. Let $\mathscr{F} \cdot \in \mathbf{D}(E)$ be the mapping cone of the map $m$ in (2). It follows that $H^{i}(E, \mathscr{F} \otimes P)=0$ for all $i$ and all $P$. Hence $\mathscr{F}=0$ and therefore $m$ is an isomorphism between vector bundles on $E$.

In summary, we have proved the following lemma.

Lemma 4.4. Assume that $M$ is a divisor on $X$ such that $a_{X *} \mathscr{O}_{X}(M)$ is an ample vector bundle on $E$ of degree $r$ and the scheme $\mathscr{Z}_{M}$ defined in (11) dominates $X$. Then we can write $|M|=\left|L_{M}\right|+\mathscr{D}_{M, 0}$ such that $|M+P|=\left|L_{M}\right|+\mathscr{D}_{M, P}$ for all $P, a_{X *} \mathscr{O}_{X}\left(\mathscr{D}_{0}\right)$ contains an ample vector bundle of degree 1 , denoted $\mathscr{E}_{M}^{+}$, and $a_{X *} \mathscr{O}_{X}\left(L_{M}\right)$ contains $\mathscr{E}_{M}^{0}=$ $\mathscr{O}_{E}^{\oplus h^{0}(M)}$.

Furthermore, we have an isomorphism of vector bundles

$$
m: \mathscr{E}_{M}^{0} \otimes \mathscr{E}_{M}^{+}=\left(\mathscr{O}_{E}^{\oplus h^{0}(X, M)}\right) \otimes \mathscr{E}_{M}^{+} \stackrel{\sim}{\rightarrow} a_{X *} \mathscr{O}_{X}(M) .
$$

This implies, in particular, that

$$
h^{0}(X, M) \cdot \operatorname{rk}\left(\mathscr{E}_{M}^{+}\right)=h^{0}\left(F,\left.M\right|_{F}\right) .
$$

Definition 4.5. We call the decomposition $|M|=\left|L_{M}\right|+\mathscr{D}_{M, 0}$ in Lemma 4.4 a uniform decomposition of $|M|$, in the sense that the decomposition $|M+P|=\left|L_{M}\right|+\mathscr{D}_{M, P}$ holds for all $P$ uniformly. Moreover, $a_{*} \mathscr{O}_{X}(M) \cong \mathscr{E}_{M}^{0} \otimes \mathscr{E}_{M}^{+}$is called a uniform decomposition of $a_{*} \mathscr{O}_{X}(M)$.

It is possible to have further decomposition. Let $B$ be a fixed divisor of $\left|L_{M}\right|$ and $L_{M}^{\prime}=L_{M}-B$. That is, $\left|L_{M}\right|=\left|L_{M}^{\prime}\right|+B$. Since one has $V^{0}\left(L_{M}^{\prime}\right) \subset V^{0}\left(L_{M}\right) \neq \widehat{E}$, and $h^{0}\left(L_{M}^{\prime}\right)=h^{0}\left(L_{M}\right)$, it follows that $\mathscr{E}_{M}^{0} \subset a_{*} \mathscr{O}_{X}\left(L_{M}^{\prime}\right) \subset a_{*} \mathscr{O}_{X}\left(L_{M}\right)$. Moreover,

$$
\mathscr{E}_{M}^{0} \otimes \mathscr{E}_{M}^{+} \stackrel{\cdot B}{\longrightarrow} a_{*} \mathscr{O}_{X}(M)
$$

is an isomorphism. 
Lemma 4.6. Given a divisor $M$ on $X$ such that $a_{*} \mathscr{O}_{X}(M)$ is ample and $\mathscr{Z}_{M}$ dominates $X$. After replacing $X$ by its birational model and replacing $M$ by its total transform, there exists a uniform decomposition $|M|=\left|L_{M}\right|+\mathscr{D}_{M, 0}+E_{M}$ such that $L_{M}$ is base point free and $\mathscr{D}_{M, 0}$ is nef and smooth. Moreover, there is an isomorphism of vector bundles

$$
\mathscr{E}_{M}^{0} \otimes \mathscr{E}_{M}^{+} \stackrel{\cdot E_{M}}{\longrightarrow} a_{*} \mathscr{O}_{X}(M) .
$$

This decomposition is called a refined uniform decomposition. The induced isomorphism of vector bundles is called a refined uniform decomposition of $a_{*} \mathscr{O}_{X}(M)$.

Proof. Consider $\pi: \widetilde{X} \rightarrow X$ a birational morphism resolving the base loci of $\left|L_{M}\right|$ and resolving $\cap_{P \in \widehat{E}} \mathscr{D}_{M, P}$. Let $\widetilde{L}_{M}$ and $\widetilde{\mathscr{D}}_{M, P}$ be the proper transform of $L_{M}$ and $\mathscr{D}_{M, P}$ respectively. Note that for any curve $C$, if $C \subset \operatorname{Supp}\left(\widetilde{\mathscr{D}}_{M, P}\right)$, then $C \not \subset \operatorname{Supp}\left(\widetilde{\mathscr{D}}_{M, Q}\right)$ for general $Q$. Hence $C \cdot\left(\widetilde{\mathscr{D}}_{M, P}\right)=C \cdot\left(\widetilde{\mathscr{D}}_{M, Q}\right) \geq 0$, that is $\widetilde{\mathscr{D}}_{M, P}$ is nef.

Similarly, one can show that there exists $\widetilde{\mathscr{E}}_{M}^{0}, \widetilde{\mathscr{E}}_{M}^{+}$subbundles of $a_{*} \pi_{*} \mathscr{O}_{\widetilde{X}}\left(\widetilde{L}_{M}\right)$ and $a_{*} \pi_{*} \mathscr{O}_{\widetilde{X}}\left(\widetilde{\mathscr{D}}_{M, 0}\right)$ respectively such that

$$
\widetilde{\mathscr{E}}_{M}^{0} \otimes \widetilde{\mathscr{E}}_{M}^{+} \hookrightarrow a_{*} \mathscr{O}_{\widetilde{X}}\left(\widetilde{L}_{M}\right) \otimes a_{*} \mathscr{O}_{\widetilde{X}}\left(\widetilde{\mathscr{D}}_{M, 0}\right) \stackrel{\cdot E_{M}}{\longrightarrow} a_{*} \mathscr{O}_{\widetilde{X}}\left(\pi^{*} M\right)=a_{*} \mathscr{O}_{X}(M)
$$

is an isomorphism. Note that $h^{0}\left(\widetilde{X}, \widetilde{L}_{M}\right)=h^{0}\left(X, L_{M}\right)=h^{0}(X, M)$, hence $\widetilde{\mathscr{E}}_{M}^{0}=\mathscr{E}_{M}^{0}$ and therefore $\widetilde{\mathscr{E}}_{M}^{+} \cong \mathscr{E}_{M}^{+}$.

We will need the following facts.

Lemma 4.7. Let $L$ be a line bundle on a smooth projective variety. Assume that $L=L_{1} \otimes L_{2}$ is the tensor product of two line bundles and there exists subspaces $W_{1} \subset H^{0}\left(L_{1}\right)$ and $W_{2} \subset H^{0}\left(L_{2}\right)$ such that the natural map $W_{1} \otimes W_{2} \rightarrow H^{0}(L)$ is an isomorphism. Then the following holds.

(1) If $L$ is free, then both $L_{1}$ and $L_{2}$ are free.

(2) If $\operatorname{dim} W_{1}=1$, then $W_{1}=H^{0}\left(L_{1}\right), W_{2}=H^{0}\left(L_{2}\right)$ and $|L|=$ $\left|L_{2}\right|+D_{1}$, where $D_{1}$ is the unique effective divisor in $\left|L_{1}\right|$.

Proof. (1). Let $s_{1}, \ldots s_{n}$ and $t_{1}, \ldots t_{m}$ be basis of $W_{1}, W_{2}$ respectively. Then $\left\{s_{i} t_{j}\right\}$ forms a basis of $H^{0}(L)$. Suppose that $s_{1}, \ldots s_{n}$ has a common zero at $x \in X$, then $x$ is a common zero of $\left\{s_{i} t_{j}\right\}$ for all $i, j$, which is absurd. Hence $W_{1}$ is free and so is $L_{1}$. The same argument holds for $W_{2}$ and $L_{2}$.

(2). Since $\operatorname{dim} W_{1}=1$, we then have $h^{0}(L)=\operatorname{dim} W_{2}$. On the other hand, $h^{0}(L) \geq h^{0}\left(L_{2}\right) \geq \operatorname{dim} W_{2}$. Hence $W_{2}=H^{0}\left(L_{2}\right)$. Let $0 \neq s \in W_{1}$ and let $D$ be the divisor $\operatorname{div}(s)$. Then $W_{1} \otimes W_{2}=H^{0}(L)$ 
implies that $D$ is a fixed divisor of the linear system $|L|$. In particular, $h^{0}\left(L_{1}\right)=h^{0}(D)=1$.

Corollary 4.8. Apply the above Lemma to a refined uniform decomposition $|M|=L_{M}+\mathscr{D}_{M, 0}+E_{M}$, one has the following:

(1) If $\operatorname{rk}\left(\mathscr{E}_{M}^{+}\right)=1$ or $\operatorname{rk}\left(\mathscr{E}_{M}^{0}\right)=1$, then $\mathscr{E}_{M}^{+}=\mathscr{V}_{M}^{+}$and $\mathscr{E}_{M}^{0}=\mathscr{V}_{M}^{0}$.

(2) If $r k\left(\mathscr{V}_{M}^{0}\right)=1$, then $\operatorname{rk}\left(\mathscr{E}^{0}\right)=1$ and hence $h^{0}(X, M)=1$.

(3) Let $L_{M, F}$ and $\mathscr{D}_{M, F}$ denote the restriction of $L_{M}$ and $\mathscr{D}_{M, 0}$ to general fiber $F$ respectively. If $\left|M_{F}\right|$ is free, then both $\left|L_{M, F}\right|$ and $\left|\mathscr{D}_{M, F}\right|$ are free,

(4) If $r k\left(\mathscr{E}_{M}^{+}\right)=1$, then $\mathscr{D}_{M, 0} \equiv F$ for a general fiber $F$. Moreover, the map restricting to general fiber $H^{0}(X, M) \rightarrow H^{0}\left(F, M_{F}\right)$ is surjective.

Proof. The first three statements are clear. We prove the last statement. If $\mathscr{E}_{M}^{+}$is a line bundle of degree 1 , denoted $\mathscr{O}_{E}(p)$ for some $p \in E$. Then $\mathscr{O}_{E}(p) \subset a_{*} \mathscr{O}_{X}\left(\mathscr{D}_{M, 0}\right)$ and hence $a_{X}^{*} \mathscr{O}_{E}(p) \otimes P \subset \mathscr{D}_{M, P}$ for all $P$. For general $P, \mathscr{D}_{M, P}$ is irreducible containing the fiber $F$ such that $\mathscr{O}_{X}(F)=a_{X}^{*} \mathscr{O}_{E}(p) \otimes P$. In particular, $\mathscr{D}_{M, 0} \equiv F$.

Now $a_{X *} \mathscr{O}_{X}(M)=\left(\mathscr{O}_{E}^{\oplus h^{0}(X, M)}\right) \otimes \mathscr{E}_{M}^{+}=\mathscr{E}_{M}^{+} h^{0}(X, M)$, which is clearly generically generated by global sections. In particular, the map restricting to general fiber $H^{0}(X, M) \rightarrow H^{0}\left(F, M_{F}\right)$ is surjective.

Lemma 4.9. Keep the same assumption as that of Lemma 4.4. If $M=M_{1}+M_{2}$ such that $a_{X *} \mathscr{O}_{X}\left(M_{1}\right)$ is a non-trivial nef vector bundle and $a_{X *} \mathscr{O}_{X}\left(M_{2}\right)$ is an ample vector bundle. Let $M=L_{M}+\mathscr{D}_{M, 0}$ be a uniform decomposition. Then the following holds.

(1) There exists an element $Q_{0} \in \operatorname{Pic}^{0}(E)$ such that $V^{0}\left(M_{1}\right)=$ $\left\{Q_{0}\right\}$

(2) $M_{2}-Q_{0}$ admits a uniform decomposition $M_{2}-Q_{0}=L_{M_{2}}+\mathscr{D}_{M, 0}$ such that $\left|M_{2}-Q_{0}+P\right|=\left|L_{M_{2}}\right|+\mathscr{D}_{M, P}$ for all $P \in \widehat{E}$;

(3) $a_{X *} \mathscr{O}_{X}\left(M_{2}-Q_{0}\right)=\mathscr{E}_{M_{2}}^{0} \otimes \mathscr{E}_{M}^{+}$, where $\mathscr{E}_{M_{2}}^{0}=\mathscr{O}_{E}^{\oplus h^{0}\left(X, M_{2}\right)}$;

(4) $h^{0}\left(X, M_{2}\right) \cdot r k\left(\mathscr{E}_{M}^{+}\right)=h^{0}\left(F, M_{2, F}\right)$, where $M_{2, F}$ is the restriction of $M_{2}$ to the general fiber $F$.

(5) $L_{M}=L_{M_{2}}+Q_{0}+M_{1}$ and the restriction to $F$ yields $L_{M, F}=$ $L_{M_{2}, F}+M_{1, F}$.

Proof. Since $a_{X *} \mathscr{O}_{X}\left(M_{1}\right)$ is a non-trivial nef vector bundle, there exists $Q_{0} \in \widehat{E}$ such that $H^{0}\left(X, \mathscr{O}_{X}\left(M_{1}\right) \otimes Q_{0}\right) \neq 0$ and we fix a divisor $G_{Q_{0}} \in$ $\left|M_{1}+Q_{0}\right|$. We have $G_{Q_{0}}+\left|M_{2}-Q_{0}+P\right| \subset|M+P|=\left|L_{M}\right|+\mathscr{D}_{M, P}$. Since $\mathscr{D}_{M, P}$ is irreducible and $\mathscr{D}_{M}$ dominates $X$, therefore for general $P, \mathscr{D}_{M, P} \nsubseteq \operatorname{Supp}\left(G_{Q_{0}}\right)$ and hence $\mathscr{D}_{M, P}$ is a fixed part of the linear system $\left|M_{2}-Q_{0}+P\right|$. Since $h^{0}\left(X, \mathscr{O}_{X}\left(M_{2}\right) \otimes P\right)$ is constant for every 
$P \in \widehat{E}, \mathscr{D}_{P}$ is a fixed part for all $\left|M_{2}-Q_{0}+P\right|$. By the similar argument as in Lemma 4.4, we see the desired structure of $a_{X *} \mathscr{O}_{X}\left(M_{2}-Q_{0}\right)$.

If there exists $Q_{1} \in V^{0}\left(M_{1}\right)$ different from $Q_{0}$, then by the same argument we conclude that $\mathscr{D}_{M, P}$ is a fixed divisor of $\left|M_{2}-Q_{1}+P\right|$ for $P \in \widehat{E}$. We then conclude that the Abel-Jacobi map for the fixed divisors of $\left|M_{2}+P\right|$ is not the identity of $\widehat{E}$, which contradicts Lemma 4.3. Hence $V^{0}\left(M_{1}\right)=\left\{Q_{0}\right\}$.

In the rest of this article, we focus on the following threefolds.

Definition 4.10. A threefold $X$ is said to be a special irregular threefold, if $X$ is a threefold of general type satisfying Condition $(\dagger)$, i,e, $\chi\left(X, \omega_{X}\right) \leq 0$ and the Albanese image is an elliptic curve, and for $x \in X$ general, both $E_{2, x}:=a_{X *}\left(\omega_{X}^{2} \otimes \mathscr{I}_{x}\right)$ and $E_{3, x}:=a_{X *}\left(\omega_{X}^{3} \otimes \mathscr{I}_{x}\right)$ are not M-regular.

We shall work on special irregular threefolds from now on.

According to Lemma 4.2, $\mathscr{Z}_{3 K_{X}}$ dominates $X$ when $X$ is a special irregular threefold. We now apply Lemma 4.4 and get a uniform decomposition $3 K_{X}=L_{3 K}+\mathscr{D}_{3 K, 0}$ with $\left|3 K_{X}\right|=\left|L_{3 K}\right|+\mathscr{D}_{3 K, 0}$ and

$$
a_{X *} \mathscr{O}_{X}\left(3 K_{X}\right)=\mathscr{E}_{3 K}^{0} \otimes \mathscr{E}_{3 K}^{+}
$$

For the general fiber $F$ of $a_{X}$, we may write the restriction as $3 K_{F}=$ $L_{3 K, F}+\mathscr{D}_{3 K, F}$. We will denote by $F_{0}$ the minimal model of $F$ and denote by $\sigma: F \rightarrow F_{0}$ the contraction morphism.

We have

$$
H^{0}\left(F, 3 K_{F}\right) \simeq W_{3}^{0} \otimes W_{3}^{+}
$$

where $W_{3}^{0}$ (resp. $W_{3}^{+}$) is the corresponding subspace of $H^{0}\left(F, L_{3 K, F}\right)$ (resp. $\left.H^{0}\left(F, \mathscr{D}_{3, F}\right)\right)$ and since $a_{X *} \mathscr{O}_{X}\left(L_{3 K}\right)$ contains $\mathscr{E}_{3 K}^{0}=\mathscr{O}_{E}^{h^{0}\left(X, 3 K_{X}\right)}$ we have

$$
h^{0}\left(F, L_{3 K, F}\right)=\operatorname{rk}\left(a_{X *} \mathscr{O}_{X}\left(L_{3}\right)\right) \geq \operatorname{rk}\left(\mathscr{E}_{3 K}^{0}\right)=P_{3}(X)>1
$$

Let $\varphi: X \rightarrow Z$ be the Stein factorization of the bi-canonical (resp. tri-canonical) map if $P_{2}(X) \geq 2$ (resp. $P_{3}(X) \geq 2$ ). Let $S$ be the general hyperplane section if $\operatorname{dim} \varphi(X) \geq 2$ or general irreducible fiber of $\varphi$ if $\operatorname{dim} \varphi(X)=1$. Then we will prove that $|5 K+P|$ is birational by studying the restriction $|5 K|_{S}$ in the remaining part of the article. 


\section{Irregular threefolds Whose Albanese fibers have $p_{g}(F) \neq 0$}

The aim of this section is to prove the main theorem for special irregular threefolds with $p_{g}(F) \neq 0$. If $p_{g}(F) \neq 0$, then $a_{*} \mathscr{O}_{X}\left(K_{X}\right)$ is non-trivial.

Considering the divisor $3 K_{X}=K_{X}+2 K_{X}$ and refined uniform decomposition $\left|3 K_{X}+P\right|=\left|L_{3 K}\right|+\mathscr{D}_{3 K, P}+E_{3}$, we may apply Lemma 4.4 and Lemma 4.9 to get:

$$
\left\{\begin{array}{l}
a_{*} \mathscr{O}_{X}\left(K_{X}\right)=\left(Q^{-1}\right)^{\oplus p_{g}(F)}, \text { for some } Q \in \widehat{E} \\
\left|2 K_{X}+P-Q\right|=\left|L_{2 K}\right|+\mathscr{D}_{3 K, P}+E_{2} \\
a_{*} \mathscr{O}_{X}\left(2 K_{X}-Q\right)=\mathscr{O}_{E}^{h^{0}\left(2 K_{X}\right)} \otimes \mathscr{E}_{3 K}^{+} \\
a_{*} \mathscr{O}_{X}\left(3 K_{X}\right)=\mathscr{O}_{E}^{h^{0}\left(3 K_{X}\right)} \otimes \mathscr{E}_{3 K}^{+}
\end{array}\right.
$$

where the first formula holds because we know that have the decomposition formula for $a_{X *} \mathscr{O}_{X}\left(K_{X}\right)$ (see the main theorem in [PPS] ) and the fact that $V^{0}\left(K_{X}\right)=\{Q\}$ by Lemma 4.9 and, as in Remark 4.6, we may also assume that $\left|L_{2 K}\right|$ is base point free. For simplicity of notations, we may also write $\mathscr{D}_{P}$ instead of $\mathscr{D}_{3 K, P}$.

As before, we denote by $L_{3 K, F}$ (resp. $L_{2 K, F}$ ) the restriction of $L_{3 K}$ (resp. $L_{2 K}$ ) to a general fiber $F$ of $a_{X}$. We note that $\mathscr{D}_{P_{1}, F} \simeq \mathscr{D}_{P_{2}, F}$ for $P_{1}, P_{2} \in \operatorname{Pic}^{0}(E)$ and we denote by $\mathscr{D}_{F}$ this divisor.

We know that the natural map

$$
W_{3}^{0} \otimes W_{3}^{+} \stackrel{\cdot E_{3}}{\longrightarrow} H^{0}\left(F, 3 K_{F}\right)
$$

is an isomorphism, where $W_{3}^{0}$ and $W_{3}^{+}$defined as in the previous section. Moreover, let $W_{2}^{0}:=\mathscr{O}_{E}^{h^{0}\left(2 K_{X}\right)} \otimes k(x) \subset H^{0}\left(F, L_{2 K, F}\right)$, where $x=a(F)$. Then we also have the natural isomorphism

$$
W_{2}^{0} \otimes W_{3}^{+} \stackrel{\cdot E_{2}}{\longrightarrow} H^{0}\left(F, 2 K_{F}\right) .
$$

We summarize some important corollaries of Lemma 4.4 and Lemma 4.9, which will be used in this section.

Lemma 5.1. Let $\sigma_{F}: F \rightarrow F_{0}$ be the contraction map to the minimal model of $F$. Suppose that $\left|m K_{F_{0}}\right|$ is base-point-free for $m=2$ or 3, then $L_{m, F}+\mathscr{D}_{F}=m \sigma^{*} K_{F_{0}}$. Moreover, $\left|L_{m, F}\right|$ and $\left|\mathscr{D}_{F}\right|$ are base point free.

Furthermore, let $\Lambda_{m}=\sigma_{F *}\left(L_{m, F}\right)$ and $\Delta=\sigma_{F *}\left(\mathscr{D}_{F}\right)$. Then both are base point free and $L_{m, F}=\sigma_{F}^{*}\left(\Lambda_{m}\right), \mathscr{D}_{F}=\sigma_{F}^{*}(\Delta)$.

Proof. Let $E:=K_{F / F_{0}}$. Then $\left|m K_{F}\right|=\sigma^{*}\left|m K_{F_{0}}\right|+m E$. From the isomorphism (3, 4), we conclude that $L_{m, F}+\mathscr{D}_{F}=m \sigma^{*} K_{F_{0}}$. Moreover, $\left|L_{m, F}\right|$ and $\left|\mathscr{D}_{F}\right|$ are base point free by Lemma 4.7.1. Hence $0 \leq L_{m, F}$. 
$E \leq m \sigma^{*} K_{F_{0}} \cdot E=0$ and $0 \leq \mathscr{D}_{F} \cdot E \leq m \sigma^{*} K_{F_{0}} \cdot E=0$. Then it is clear that $L_{m, F}=\sigma_{F}^{*}\left(\Lambda_{m}\right), \mathscr{D}_{F}=\sigma_{F}^{*}(\Delta)$.

Corollary 5.2. If $p_{g}(F)>0$, then $P_{2}(X)=h^{0}\left(F_{0}, \Lambda_{2}\right)>1$.

Proof. Note that $P_{2}(X)=\operatorname{rk}\left(\mathscr{E}_{M_{2}}\right)$ and $h^{0}\left(F_{0}, \Lambda_{2}\right)=h^{0}\left(F, L_{2, F}\right) \geq$ $\operatorname{rk}\left(\mathscr{E}_{M_{2}}\right)$. Hence it suffices to show that $\operatorname{rk}\left(\mathscr{E}_{M_{2}}\right)>1$.

Suppose on the contrary that $\operatorname{rk}\left(\mathscr{E}_{M_{2}}^{0}\right)=\operatorname{dim} W_{2}^{0}=1$. Then by Corollary 4.7,2, one has $h^{0}\left(F, L_{2, F}\right)=h^{0}\left(F_{0}, \Lambda_{2}\right)=1$. Moreover, $\left|2 K_{F_{0}}\right|=\Lambda_{2}+|\Delta|$. Since $\left|2 K_{F_{0}}\right|$ is base point free by Proposition 2.2. $\Lambda_{2}=0$ and hence so is $L_{2, F}$. Now Lemma 4.9.5 gives $L_{3, F}=$ $L_{2, F}+K_{F}=K_{F}$. Hence $W_{3}^{0} \subset H^{0}\left(F, L_{3 K, F}\right) \simeq H^{0}\left(F, K_{F}\right)$. Also, $\left|2 K_{F_{0}}\right|=|\Delta|$ and $\mathscr{D}_{F}=\sigma^{*}\left(2 K_{F_{0}}\right)$ and hence $W_{3}^{+}=H^{0}\left(F, \mathscr{D}_{3 K, F}\right) \simeq$ $H^{0}\left(F_{0}, 2 K_{F_{0}}\right)$. It follows that the isomorphism

$$
\Phi_{W}: W_{3}^{0} \otimes W_{3}^{+} \cong W_{3}^{0} \otimes H^{0}\left(F, 2 K_{F}\right) \cong H^{0}\left(F, 3 K_{F}\right)
$$

is compatible with $\Phi: H^{0}\left(K_{F}\right) \otimes H^{0}\left(2 K_{F}\right) \rightarrow H^{0}\left(3 K_{F}\right)$. For any subspace $W \subset H^{0}\left(K_{F}\right)$ of dimension $\geq 2$, it is obvious that the induced map $\left.\Phi\right|_{W \otimes H^{0}\left(2 K_{F}\right)}: W \otimes H^{0}\left(2 K_{F}\right) \rightarrow H^{0}\left(3 K_{F}\right)$ can not be injective. Therefore, $\operatorname{dim} W_{3}^{0}=1$ and hence $h^{0}\left(2 K_{F}\right)=h^{0}\left(3 K_{F}\right)$, which is the desired contradiction.

The rest of this section is devoted to the proof of the following theorem.

Theorem 5.3. Let $X$ be a special irregular 3 -fold. Then $\left|5 K_{X}\right|$ induces a birational map of $X$ if any one of the following conditions holds

(1) $p_{g}(F)>0$;

(2) $P_{2}(X) \geq 2$ and $K_{F_{0}}^{2} \geq 3$.

Proof. Let $\varphi: X \rightarrow \mathbb{P}^{a}$ be the morphism induced by $\left|L_{2 K}\right|$ and let $S$ be an irreducible component of a general pencil of $\left|L_{2 K}\right|$. It is clear that $S$ is a smooth of general type. We first claim that the induced map $S \rightarrow E$ is surjective. To see the claim, suppose on the contrary that $S \rightarrow E$ is not surjective, then $S=F$ and hence $L_{2 K}=F$ (resp. $\left.L_{3 K}\right)$. It follows that $V^{0}\left(X, L_{2 K}\right)=\widehat{E}$. This is a contradiction since $V^{0}\left(X, L_{2 K}\right) \neq \widehat{E}$ by Lemma 4.3 .

We will also use the following notation: let $V$ be a $\mathbb{Q}$-divisor on $X$ such that $S \nsubseteq \operatorname{Supp}(V)$, then we denote by $V_{S}$ the restriction of $V$ on $S$.

We denote by $\pi: X \rightarrow X_{0}$ the morphism from $X$ to its canonical model $X_{0}$ and let $\sigma_{S}: S \rightarrow S_{0}$ be the contraction to its minimal model. Then $\pi^{*} K_{X_{0}}$ is a $\mathbb{Q}$-Cartier nef and big divisor on $X$. We then consider 
the refined uniform decomposition $\left|2 K_{X}-Q\right|=\left|L_{2 K}\right|+\mathscr{D}_{2 K, 0}+E_{2}$ and the short exact sequence:

$$
\begin{gathered}
0 \rightarrow \mathscr{O}_{X}\left(K_{X}+\left\lceil 2 K_{X}+2 \pi^{*} K_{X_{0}}-E_{2}\right\rceil-S\right) \rightarrow \mathscr{O}_{X}\left(K_{X}+\left\lceil 2 K_{X}+2 \pi^{*} K_{X_{0}}-E_{2}\right\rceil\right) \\
\rightarrow \mathscr{O}_{S}\left(\left(K_{X}+\left\lceil 2 K_{X}+2 \pi^{*} K_{X_{0}}-E_{2}\right\rceil\right)_{S}\right) \rightarrow 0 .
\end{gathered}
$$

Note that $2 K_{X}-S-E_{2} \sim \mathscr{D}_{2 K, Q}=$ : $\mathscr{D}_{Q}$ is nef and $\pi^{*} K_{X_{0}}$ is nef and big. Thus, by Kawamata-Viehweg vanishing theorem, we have the surjective map

$H^{0}\left(X, \mathscr{O}_{X}\left(K_{X}+\left\lceil 2 K_{X}+2 \pi^{*} K_{X_{0}}-E_{2}\right\rceil\right)\right) \rightarrow H^{0}\left(S, K_{S}+\left\lceil 2 \pi^{*} K_{X_{0}}+\mathscr{D}_{Q}\right\rceil_{S}\right)$.

We also know that $5 K_{X} \succeq K_{X}+\left\lceil 2 K_{X}+2 \pi^{*} K_{X_{0}}-E_{2}\right\rceil$ and $5 K_{X}-L_{2 K}$ are effective divisors. Hence in order to conclude the proof of Theorem 5.3. we just need to show that under the respective assumptions, the global section of $K_{S}+\left\lceil 2 \pi^{*} K_{X_{0}}+\mathscr{D}_{Q}\right\rceil_{S}$ induces a birational map of $S$.

By the extension theorem (see the main theorem of $[\mathrm{K}]$ ), we know that the restriction map $H^{0}\left(X, m\left(K_{X}+L_{2 K}\right)\right) \rightarrow H^{0}\left(S, m K_{S}\right)$ is surjective for any $m \geq 2$. We may take $m$ sufficient large and divisible and conclude that $\operatorname{Mov}\left(\left|m\left(K_{X}+L_{2 K}\right)\right|\right)_{S} \succeq m \sigma_{S}^{*} K_{S_{0}}$, where $\operatorname{Mov}\left(\mid m\left(K_{X}+\right.\right.$ $\left.\left.L_{2 K}\right) \mid\right)$ denotes the moving part of the linear system $\left|m\left(K_{X}+L_{2 K}\right)\right|$. Since $\mathscr{D}_{P}$ is nef and contains no base divisor for each $P \in \operatorname{Pic}^{0}(E)$, we have $\operatorname{Mov}\left|m \mathscr{D}_{Q}\right|=\left|m \mathscr{D}_{Q}\right|$ for $m \geq 2$ and hence

$\operatorname{Mov}\left(\left|m\left(K_{X}+L_{2 K}\right)\right|\right)+m \mathscr{D}_{Q} \preceq \operatorname{Mov}\left(\mid m\left(K_{X}+L_{2 K}+\mathscr{D}_{Q} \mid\right) \preceq 3 m \pi^{*} K_{X_{0}}\right.$.

Thus,

$$
\sigma_{S}^{*} K_{S_{0}}+\mathscr{D}_{Q, S} \preceq \operatorname{Mov}\left(\left|m\left(K_{X}+L_{2 K}\right)\right|\right)_{S}+\mathscr{D}_{Q, S} \preceq 3 \pi^{*} K_{X_{0}, S}
$$

and hence

$$
2 \pi^{*} K_{X_{0}, S}+\mathscr{D}_{Q, S} \succeq \frac{2}{3} \sigma_{S}^{*} K_{S_{0}}+\frac{5}{3} \mathscr{D}_{Q, S}
$$

We then apply Theorem 2.3 to show that the global section of $K_{S}+$ $\left\lceil 2 \pi^{*} K_{X_{0}}+\mathscr{D}_{Q}\right\rceil_{S}$ induces a birational map of $S$. We distinguish two cases depending on whether $\mathscr{D}_{P}$ is a fiber of $a_{X}$ or not for $P$ general.

Case 1. $\mathscr{D}_{P}$ is a general fiber of $a_{X}$.

In this case, $F=\mathscr{D}_{P}$ is a general fiber of $a_{X}$ and hence $\mathscr{D}_{P, F}=0$. We regard $\Gamma:=F \cap S$ as a general fiber of $S \rightarrow E$ or a general member of the linear system $\left|L_{2 K, F}\right|$ on $F$. Note that the morphism $S \rightarrow E$ factors through its minimal model $S_{0}$, hence $\Gamma \cdot K_{S}=\Gamma \cdot \sigma_{S}^{*} K_{S_{0}}$. Thus considering $\Gamma$ as a curve on $S$ yields

$$
\sigma_{S}^{*} K_{S_{0}} \cdot \mathscr{D}_{Q, S}=\sigma_{S}^{*} K_{S_{0}} \cdot \Gamma=K_{S} \cdot \Gamma=2 g(\Gamma)-2 .
$$


On the other hand, considering $\Gamma$ as a curve on $F$ leads to

$$
2 g(\Gamma)-2=\left.\left(K_{F}+\left.S\right|_{F}\right) \cdot S\right|_{F} .
$$

Claim 1.1. $\sigma_{S}^{*} K_{S_{0}} \cdot \mathscr{D}_{P, S} \geq 6$ and hence $\left(\frac{2}{3} \sigma_{S}^{*} K_{S_{0}}+\frac{5}{3} \mathscr{D}_{P, S}\right)^{2} \geq \frac{40}{3}$.

If $\left|2 K_{F_{0}}\right|$ is base point free (which is the case when $p_{g}(F) \neq 0$ or $K_{F_{0}}^{2} \geq 5$ by [BPV, Theorem 5.1 and Theorem 7.4]), then $L_{2 K, F}=2 \sigma_{F}^{*} K_{F_{0}}$ by Lemma 5.1. It follows that

$$
\sigma_{S}^{*} K_{S_{0}} \cdot \mathscr{D}_{Q, S}=2 g(\Gamma)-2=\left(K_{F}+\sigma_{F}^{*} 2 K_{F_{0}}\right) \cdot \sigma_{F}^{*} 2 K_{F_{0}}=6 K_{F_{0}}^{2} \geq 6
$$

If $\left|2 K_{F_{0}}\right|$ has base points (which could happen when $K_{F_{0}}^{2}=3$ or 4 ), we note that still have $\left|2 K_{F}\right|=\left|L_{2 K}\right|_{F}+E_{2, F}$. By the assumption that $K_{F_{0}}^{2} \geq 3$ and the main result of [X2], we know that $\left|L_{2 K}\right|$ induces a generically finite morphism of $F$ and $h^{0}\left(F, L_{2 K, F}\right)=P_{2}(F) \geq 4$. It is easy to see that $\left(L_{2 K, F}\right)^{2} \geq 2\left(P_{2}(F)-2\right) \geq 4$. Hence $K_{F} \cdot L_{2 K, F} \geq$ $\sigma_{F}^{*} K_{F_{0}} \cdot L_{2 K, F} \geq 4$ by Hodge index theorem. Thus $\sigma_{S}^{*} K_{S_{0}} \cdot \mathscr{D}_{P, S}=$ $\left.\left(K_{F}+\left.S\right|_{F}\right) \cdot S\right|_{F} \geq 8$.

Claim 1.2. $\left(\frac{2}{3} \sigma_{S}^{*} K_{S_{0}}+\frac{5}{3} \mathscr{D}_{P, S}\right) \cdot C \geq 3$ for a general curve $C$ on $S$. Suppose first that $a(C)$ is a point, then $C=\Gamma$. Therefore $\sigma_{0}^{*} K_{S_{0}} \cdot C \geq 6$ and $\left(\frac{2}{3} \sigma_{S}^{*} K_{S_{0}}+\frac{5}{3} \mathscr{D}_{P, S}\right) \cdot C \geq 4$.

If $a(C)$ is not a point, then $\mathscr{D}_{P, S} \cdot C \geq 1$. The claim holds if $\sigma_{S}^{*} K_{S_{0}}$. $C \geq 2$. If $\sigma_{S}^{*} K_{S_{0}} \cdot C=0$, one has a contradiction by Hodge Index Theorem. If $\sigma_{S}^{*} K_{S_{0}} \cdot C=1$, then Hodge Index Theorem implies that $C \equiv \sigma_{S}^{*} K_{S_{0}}$. Then Claim 1 gives $\mathscr{D}_{P, S} \cdot C \geq 6$ and hence $\left(\frac{2}{3} \sigma_{S}^{*} K_{S_{0}}+\right.$ $\left.\frac{5}{3} \mathscr{D}_{P, S}\right) \cdot C \geq \frac{32}{3}$.

Finally, we conclude by Theorem 2.3 that the global section of $K_{S}+$ $\left\lceil 2 \pi^{*} K_{X_{0}}+\mathscr{D}_{P}\right\rceil_{S}$ induces a birational map of $S$.

Case 2. $a_{X *} \mathscr{D}_{P}$ has rank $>1$.

We start with the following observation.

Claim 2.1. $\mathscr{D}_{P, S}$ is nef and big. Moreover, $\mathscr{D}_{P, S}^{2} \geq 2$.

Proof. We already know that $\mathscr{D}_{P}$ is nef. It suffices to see that $\mathscr{D}_{P, S}$ is big. First note that $\mathscr{D}_{P} \equiv \mathscr{D}_{Q}$ for $P, Q \in \operatorname{Pic}^{0}(X)$ and $S \rightarrow E$ is a surjective morphism. Hence the Iitaka model of $\mathscr{D}_{P}$ dominates $E$ (and so does $\left.\mathscr{D}_{P, S}\right)$.

Suppose that $\mathscr{D}_{P, S}$ is not big, then it is the pull-back on $S$ of an effective divisor of $E$. We may write $\mathscr{D}_{P, S}=a^{*} N$ for some ample divisor $N$. Let $C$ be connected component of $S \cap F$. One has that $g(C) \geq 2$ since $S$ is of general type and the restriction $\mathscr{D}_{P, C}=0$.

We then consider the short exact sequence

$$
0 \rightarrow \mathscr{O}_{X}\left(\mathscr{D}_{P}-S\right) \rightarrow \mathscr{O}_{X}\left(\mathscr{D}_{P}\right) \rightarrow \mathscr{O}_{S}\left(\mathscr{D}_{P, S}\right) \rightarrow 0
$$


It follows from $\operatorname{rk}\left(a_{*} \mathscr{D}_{P}\right)>1$ that $a_{X *} \mathscr{O}_{X}\left(\mathscr{D}_{P}-S\right) \neq 0$ and hence $\mathscr{D}_{P, F} \geq\left. S\right|_{F}$. Further restriction to $C$ yields $0=\mathscr{D}_{P, C} \geq\left. S\right|_{C}$. However, since $2 K_{X}=\mathscr{D}_{P}+S+E_{2}, 2 K_{X}+2 S=\mathscr{D}_{P}+3 S+E-2$. Its restriction to $S$ and further to $C$ leads to

$$
0<\left.\left(2 K_{S}\right)\right|_{C}=\mathscr{D}_{P, C}+\left.3 S\right|_{C}+\left.E_{2}\right|_{C}=0
$$

which is a contradiction.

We now prove that $\mathscr{D}_{P, S}^{2} \geq 2$. If $S$ is of maximal Albanese dimension, we know by [BPS, v5, Theorem 6.7] that $\operatorname{vol}\left(S, \mathscr{D}_{P, S}\right)=\mathscr{D}_{P, S}^{2} \geq$ $\frac{2 r}{2 r-1} h^{0}\left(S, \mathscr{D}_{P, S}+Q\right)$, where $Q \in \operatorname{Pic}^{0}(S)$ general and $r$ is the least positive integer such that $r K_{S} \succeq V$. Hence $\mathscr{D}_{P, S}^{2} \geq 2$.

If $a_{S}: S \rightarrow A_{S}$ maps onto a curve. Then we may apply Proposition 2.4 to conclude that $\mathscr{D}_{P, S}^{2} \geq 2$.

Claim 2.2. $\left(\frac{2}{3} \sigma_{S}^{*} K_{S_{0}}+\frac{5}{3} \mathscr{D}_{P, S}\right)^{2}>10$.

By Claim 1, $\left(\mathscr{D}_{P, S}\right)^{2} \geq 2$. Hence by Hodge index theorem, $\sigma_{S}^{*} K_{S_{0}}$. $\mathscr{D}_{P, S} \geq 2$ and hence

$$
\left(\frac{2}{3} \sigma_{S}^{*} K_{S_{0}}+\frac{5}{3} \mathscr{D}_{P, S}\right)^{2} \geq \frac{94}{9}>10 .
$$

Claim 2.3. $\left(\frac{2}{3} \sigma_{S}^{*} K_{S_{0}}+\frac{5}{3} \mathscr{D}_{P, S}\right) \cdot C \geq 3$ for a general curve $C$ on $S$. Let $C$ be a general curve on $S$. We know that $\mathscr{D}_{P, S} \cdot C \geq 1$ since $\mathscr{D}_{P, S}$ is big and $C$ is general. If $\sigma_{S}^{*} K_{S_{0}} \cdot C \geq 2$ then the Claim follows. If $\sigma_{S}^{*} K_{S_{0}} \cdot C=0$, one has a contradiction by Hodge Index Theorem. If $\sigma_{S}^{*} K_{S_{0}} \cdot C=1$, then Hodge Index Theorem implies that $C \equiv \sigma_{S}^{*} K_{S_{0}}$. Hence

$$
\left(\frac{2}{3} \sigma_{S}^{*} K_{S_{0}}+\frac{5}{3} \mathscr{D}_{P, S}\right) \cdot C=\left(\frac{2}{3} \sigma_{S}^{*} K_{S_{0}}+\frac{5}{3} \mathscr{D}_{P, S}\right) \cdot \sigma_{S}^{*} K_{S_{0}} \geq 4 .
$$

With these Claims, we thus then conclude that $K_{S}+\left\lceil 2 \pi^{*} K_{X_{0}}+\mathscr{D}_{P}\right\rceil_{S}$ induce a birational map of $S$.

\section{IRREGULAR THREEFOLDS WITH $p_{g}(F)=0$}

We always assume that $X$ is special. Then by Lemma 4.3, for $P \in$ $\operatorname{Pic}^{0}(X)$ general, we have

$$
\left|3 K_{X}+P\right|=\left|L_{3 K}\right|+\mathscr{D}_{3 K, P}+E_{3},
$$

and

$$
\left|2 K_{X}+P\right|=\left|L_{2 K}\right|+\mathscr{D}_{2 K, P}+E_{2} .
$$

For simplicity of notations, we write $\mathscr{D}_{P}:=\mathscr{D}_{3 K, P}$ and $T_{P}:=\mathscr{D}_{2 K, P}$. by Lemma 4.6, we may and will assume that $\left|L_{3 K}\right|$ and $\left|L_{2 K}\right|$ are base point free, $\mathscr{D}_{P}$ and $T_{P}$ are smooth nef divisors for $P$ general. 
Warning. Note that a difference from last section is that there is no reason here that $P_{2}(X)>1$ and hence $L_{2 K}$ could be $\mathscr{O}_{X}$. Moreover, there is also no reason that $\mathscr{D}_{P}$ and $T_{P}$ are algebraically equivalent.

Since $p_{g}(F)=0$, it follows that $q(F)=0$ and $\chi\left(\mathscr{O}_{F}\right)=1$. Hence $1 \leq K_{F_{0}}^{2} \leq 9$ by Bogomolov-Miyaoka-Yau inequality. It is then leads to the following table

\begin{tabular}{|l|l|l|l|l|l|l|l|l|l|}
\hline$K_{F_{0}}^{2}$ & 1 & 2 & 3 & 4 & 5 & 6 & 7 & 8 & 9 \\
\hline$P_{2}\left(F_{0}\right)=1+K_{F_{0}}^{2}$ & 2 & 3 & 4 & 5 & 6 & 7 & 8 & 9 & 10 \\
$P_{3}\left(F_{0}\right)=1+3 K_{F_{0}}^{2}$ & 4 & 7 & 10 & 13 & 16 & 19 & 22 & 25 & 28 \\
\hline
\end{tabular}

We know by Theorem 2.1 that $\left|3 K_{F_{0}}\right|$ induces a birational map of $F_{0}$ for all surfaces $F_{0}$ of general type with geometric genus 0 .

Since $p_{g}(F)=q(F)=0$, it follows that $a_{*} \omega_{X}=R^{1} a_{*} \omega_{X}=0$. Moreover, $R^{2} a_{*} \omega_{X}=\omega_{E}$ by $\left[\mathrm{Ko}\right.$, Prop. 7.6]. It follows that $\chi\left(\mathscr{O}_{X}\right)=$ $-\chi\left(\omega_{X}\right)=0$ in this section.

6.1. $\mathscr{D}_{P}$ is a general fiber of $a_{X}$. We will need following lemmas.

Lemma 6.1. If $\mathscr{D}_{P}$ is a general fiber of $a_{X}$, then $L_{3 K}$ is big and nef. Thus, let $S \in\left|L_{3 K}\right|$ be a general member, we have $q(S)=1$ and $S$ dominates $E$.

Proof. Suppose that $\mathscr{D}_{P}$ is a general fiber of $a_{X}$. Then one has that $\mathscr{V}_{3 K}^{+}=\mathscr{E}_{3 K}^{+}$is a line bundle of degree 1 . By Corollary 4.8,4., we conclude that

$$
\left|L_{3 K}\right|_{F}+E_{3, F} \cong\left|3 K_{X}\right|_{F} \simeq\left|3 K_{F}\right| \cong\left|\sigma_{F}^{*} 3 K_{F_{0}}\right|+3 K_{F / F_{0}} .
$$

As $\left|3 K_{F}\right|$ always induces a birational map of $F$, we see that $\left|L_{3 K}\right|_{F}$ induces a birational morphism of $F$ and the litaka dimension of $L_{3 K}$ is at least 2 .

If $L_{3 K}$ is not big, then $\left|L_{3 K}\right|$ induces a morphism $\varphi: X \rightarrow \Sigma$ such that the restriction $\left.\varphi\right|_{F}: F \rightarrow \Sigma$ is birational for every general fiber $F$ of $a_{X}$. This implies that $a_{X} \times \varphi: X \rightarrow E \times \Sigma$ is birational, which is absurd. Therefore, $L_{3 K}$ is nef and big.

The last assertion holds because $H^{1}\left(X, \mathscr{O}_{X}(-S)\right)=H^{2}\left(X, \mathscr{O}_{X}(-S)\right)=$ 0 due to Kawamata-Viehweg vanishing. Since $S$ is big, then it is clear that $S$ dominates $E$.

Under the assumption of the previous lemma, let $\Gamma$ be a general fiber of the morphism $S \rightarrow E$. Then as $\left|L_{3 K}\right|_{F} \simeq\left|3 K_{F}\right|, \Gamma$ is a general member of $\sigma_{F}^{*}\left|3 K_{F_{0}}\right|$. Hence by Lemma 2.5 and the remark after it, $\Gamma$ is not hyperelliptic.

Lemma 6.2. Let $S \in\left|L_{3 K}\right|$ be a general member and $C$ be a very general curve on $S$ dominating $E$. Then $C \cdot \Gamma=\operatorname{deg}\left(\left.a\right|_{C}: C \rightarrow E\right) \geq 3$. 
Proof of Lemma 6.2. As $C$ is very general, there exist an algebraic deformation $\left\{C_{t}\right\}$ of $C$ such that $\cup_{t} C_{t}$ covers an open subset of $S$. Since $S$ dominate $E$ and $q(S)=1$ by Lemma 6.1, the morphism $\operatorname{Pic}^{0}(E) \rightarrow \operatorname{Pic}^{0}(S)$ is an isogeny and it follows that $\operatorname{Pic}^{0}(S) \rightarrow \operatorname{Pic}^{0}(\Gamma)$ is trivial. Therefore, for general $t_{1}, t_{2}, \mathscr{O}_{S}\left(C_{t_{1}}-C_{t_{2}}\right) \in \operatorname{Pic}^{0}(S)$ induces $\mathscr{O}_{\Gamma}\left(C_{t_{1}}-C_{t_{2}}\right) \cong \mathscr{O}_{\Gamma}$. This implies in particular that $C_{t_{1}, \Gamma} \sim C_{t_{2}, \Gamma}$, and moreover there exist a rational map $\Psi: \Gamma \rightarrow \mathbb{P}^{1}$ with $C_{t_{i}, \Gamma}$ being fibers. Since $\Gamma$ is non-hyperelliptic by Lemma 2.5 , one sees that $C \cdot \Gamma=\operatorname{deg}\left(\left.\Psi\right|_{\Gamma}: \Gamma \rightarrow \mathbb{P}^{1}\right) \geq 3$.

Proposition 6.3. Assume that $p_{g}(F)=0, K_{F_{0}}^{2} \geq 2$ and $\mathscr{D}_{P}$ is a general fiber of $a_{X}$ for $P$ general, then $\left|5 K_{X}\right|$ induces a birational map.

Proof. The proof is similar to the first case of Theorem 5.3. We assume that $\left|L_{3 K}\right|$ is base point free and pick $S$ a general member of $\left|L_{3 K}\right|$ and consider the short exact sequence

$$
\begin{aligned}
0 \rightarrow \mathscr{O}_{X}\left(K_{X}+\left\lceil 3 K_{X}+\pi^{*} K_{X_{0}}-E_{3}\right\rceil-S\right) \rightarrow \mathscr{O}_{X}\left(K_{X}+\left\lceil 3 K_{X}+\pi^{*} K_{X_{0}}-E_{3}\right\rceil\right) \\
\rightarrow \mathscr{O}_{S}\left(\left(K_{X}+\left\lceil 3 K_{X}+\pi^{*} K_{X_{0}}-E_{3}\right\rceil\right)_{S}\right) \rightarrow 0 .
\end{aligned}
$$

As before, we need to show that $\left|K_{S}+\left\lceil\pi^{*} K_{X_{0}}+\mathscr{D}_{P}\right\rceil\right|_{S}$ defines a birational map of $S$. Moreover, we again apply the extension theorem [K] to conclude that

$$
\pi^{*} K_{X_{0}, S} \succeq \frac{1}{4}\left(\sigma_{S}^{*} K_{S_{0}}+\mathscr{D}_{P, S}\right)
$$

and hence

$$
\pi^{*} K_{X_{0}, S}+\mathscr{D}_{P, S} \succeq \frac{1}{4}\left(\sigma_{S}^{*} K_{S_{0}}+5 \mathscr{D}_{P, S}\right)=: \mathscr{M} .
$$

If $K_{F_{0}}^{2} \geq 2$, we know that $\left|3 K_{F_{0}}\right|$ is base point free ([BPV, Theorem 5.1]). Then $L_{3 K, F}=3 \sigma_{F}^{*} K_{F_{0}}$. As $\mathscr{D}_{P}$ is a general fiber of $a_{X}$, denoted $F$, and $\Gamma=\mathscr{D}_{P} \cap S$. By the similar computation as in Equation 6, 7. we have

$$
\left\{\begin{array}{l}
\sigma^{*} K_{S_{0}} \cdot \mathscr{D}_{P, S}=2 g(\Gamma)-2=12 K_{F_{0}}^{2} \geq 24 \\
\mathscr{M}^{2} \geq \frac{1}{16}\left(K_{S_{0}}^{2}+10 \sigma^{*} K_{S_{0}} \cdot \mathscr{D}_{P}\right)>15
\end{array}\right.
$$

Next we verify that $\mathscr{M} \cdot C \geq 3$ for any general curve $C$. Let $C$ be a general curve on $S$. If $a(C)$ is a point, then $C=\mathscr{D}_{P, S}$ and hence

$$
\mathscr{M} \cdot C \geq \frac{1}{4} \sigma^{*} K_{S_{0}} \cdot \mathscr{D}_{P, S} \geq 6 .
$$

If $a(C)$ is not a point, then the induced map $\left.a\right|_{C}: C \rightarrow E$ is of degree $\mathscr{D}_{P, S} \cdot C \geq 2$ because $g(C) \geq 2$. We next verify that $\sigma_{S}^{*} K_{S_{0}} \cdot C \geq 2$ to achieve $\mathscr{M} \cdot C \geq 3$. 
If $C^{2}=0$, then $\sigma_{S}^{*} K_{S_{0}} \cdot C=2 g-2 \geq 2$. If $C^{2}=1$, then $\sigma_{S}^{*} K_{S_{0}} \cdot C \geq 2$ unless $\sigma_{S}^{*} K_{S_{0}} \equiv C$ by Hodge Index Theorem. In this situation, we have $\mathscr{M} \cdot C=\mathscr{M} \cdot \sigma_{S}^{*} K_{S_{0}}>30$. If $C^{2} \geq 2$, then by Hodge Index Theorem, $\sigma_{S}^{*} K_{S_{0}} \cdot C \geq 2$.

Therefore, $\mathscr{M} \cdot C \geq 3$. Hence we conclude by Theorem 2.3 that the global sections of $K_{X}+\left\lceil 3 K_{X}+\pi^{*} K_{X_{0}}-E_{3}\right\rceil$ separates two general points which are in different fibers of $a_{X}$.

On the other hand, we know that in this case $\left|3 K_{X}+P\right|=\left|L_{3 K}\right|+$ $\mathscr{D}_{P}+E_{3}$, where $\left|L_{3 K}\right|_{F} \simeq\left|3 K_{F}\right|$. Hence $\left|5 K_{X}\right|_{F} \supset\left|3 K_{F}\right|+V$ for some fixed divisor $V$ and $\left|3 K_{F}\right|$ induces a birational map of $F$. Hence $\left|5 K_{X}\right|$ induces a birational map of $X$, when $K_{F_{0}}^{2} \geq 2$.

Corollary 6.4. Assume that $K_{F_{0}}^{2}=2,4,6$, then $\mathscr{D}_{P}$ is a general fiber of $a_{X}$. Hence $\left|5 K_{X}\right|$ induces a birational map of $X$.

Proof. When $K_{F_{0}}^{2}=2,4,6, P_{3}(F)$ is a prime number and $P_{3}(X) \geq 2$ by Lemma 2.7 and 4.4 , hence rank $\mathscr{E}_{3 K}^{+}=1$ and we know that $\mathscr{D}_{P}$ is a general fiber of $a_{X}$ by Corollary 4.8. Therefore, $\left|5 K_{X}\right|$ is birational by Proposition 6.3

Proposition 6.5. Assume that $K_{F_{0}}^{2}=1$, then $\mathscr{D}_{P}$ is a general fiber of $a_{X}$. Moreover, $\left|5 K_{X}\right|$ induces a birational map of $X$.

Proof. This case is more involved.

Step 1. $P_{3}(X)=4$ and $\mathscr{D}_{P}$ is a general fiber of $a_{X}$.

If $K_{F_{0}}^{2}=1$, then $P_{3}(F)=4$ and hence $P_{3}(X)=2$ or 4 since $P_{3}(X)>1$. Suppose on the contrary that $P_{3}(X)=2$. Then $H^{0}\left(F, 3 K_{F}\right) \cong W_{3}^{0} \otimes$ $W_{3}^{+}$, where both $W_{3}^{0}$ and $W_{3}^{+}$are 2-dimensional (cf. Corollary 4.8). Then the image of $\left|3 K_{F}\right|$ is a smooth quadric in $\mathbb{P}^{3}$, which contradicts the fact that $\left|3 K_{F}\right|$ induces a birational map of $F$.

Therefore $P_{3}(X)=4$. It follows that rank $\mathscr{E}_{3 K}^{+}=1$ by Lemma 4.4 and hence $\mathscr{D}_{P}$ is a general fiber of $a_{X}$ by Lemma 4.8.

Step 2. $L_{3 K}^{3} \geq 2$, and $\mathscr{D}_{P} \cdot L_{3 K}^{2} \geq 7$. Moreover, a general section of $\left|L_{3 K, F}\right|$ is a smooth curve of genus 7 and hence $\sigma_{S}^{*} K_{S_{0}} \cdot \mathscr{D}_{P, S}=$ $K_{S} \cdot \mathscr{D}_{P, S}=12$.

The linear system $\left|L_{3 K}\right|$ defines a generically finite morphism from $X$ to $\mathbb{P}^{3}$. Hence $L_{3 K}^{3} \geq 2$.

We know that $\left|3 K_{F_{0}}\right|$ has no fixed component and has less than 2 simple base points (see for instance [Cat, Corollary 12]). Hence $\left(L_{3 K, F}\right)^{2} \geq 9-2=7$. A general member of $\left|3 K_{F_{0}}\right|$ is already smooth with genus 7 . Hence a general member $\Gamma \in\left|L_{3 K}\right|_{F}$, which is a proper transform of a general member in $\left|3 K_{F_{0}}\right|$ has genus 7 . Notice that 
$\left.a\right|_{S}: S \rightarrow E$ factor through $S_{0}$. Therefore, the exceptional set of $\sigma_{S}: S \rightarrow S_{0}$ does not intersect $F$ and hence $\Gamma$. If follows that

$$
12=2 g(\Gamma)-2=K_{S} \cdot \Gamma=\sigma_{S}^{*} K_{S_{0}} \cdot \Gamma=\sigma_{S}^{*} K_{S_{0}} \cdot \mathscr{D}_{P, S} .
$$

Step 3. $K_{S_{0}}^{2} \geq 10$.

Note that $\left(K_{X}+L_{3 K}\right)_{S}=K_{S}$. Thus $\sigma^{*} K_{S_{0}} \succeq\left(\pi^{*} K_{X_{0}}\right)_{S}+L_{3 K, S}$. Hence

$$
K_{S_{0}}^{2} \geq L_{3 K}^{3}+2 \pi^{*} K_{X_{0}} \cdot L_{3 K}^{2}+\left(\pi^{*} K_{X_{0}}\right)^{2} \cdot L_{3 K}
$$

Moreover since $\pi^{*} K_{X_{0}} \succeq \frac{1}{3}\left(\mathscr{D}_{P}+L_{3 K}\right)$, we have by Lemma 6.1 that

$$
\pi^{*} K_{X_{0}} \cdot L_{3 K}^{2} \geq \frac{1}{3} L_{3 K}^{3}+\frac{1}{3} \mathscr{D}_{P} \cdot L_{3 K}^{2} \geq 3
$$

and

$$
\begin{aligned}
\left(\pi^{*} K_{X_{0}}\right)^{2} \cdot L_{3 K} & \geq \frac{1}{3} \mathscr{D}_{P} \cdot \pi^{*} K_{X_{0}} \cdot L_{3 K}+\frac{1}{3} \pi^{*} K_{X_{0}} \cdot L_{3 K}^{2} \\
& \geq \frac{1}{3} \mathscr{D}_{P} \cdot \pi^{*} K_{X_{0}} \cdot L_{3 K}+1 .
\end{aligned}
$$

Note that $a_{X}$ factors through $\pi: X \rightarrow X_{0}$. Moreover, for any $m$ sufficiently large and divisible and $P \in \operatorname{Pic}^{0}(E)_{\text {tor }}$ torsion, $\left|m K_{X}+P\right|=$ $\pi^{*}\left|m K_{X_{0}}+P\right|+m K_{X / X_{0}}$. Moreover, for $m \geq 2, a_{X *} \omega_{X}^{m}$ is $\mathrm{IT}^{0}$ sheaves. Hence $a_{X *} \omega_{X}^{m}$ is continuously globally generated by [PP]. Thus the evaluation map

$$
\bigoplus_{P \in \operatorname{Pic}^{0}(E)_{\text {tor }}} H^{0}\left(m K_{X}+P\right) \rightarrow H^{0}\left(F, m K_{F}\right)
$$

is surjective. We then conclude that $\pi^{*} K_{X_{0}, F}=\sigma^{*} K_{F_{0}}$. Thus

$$
\pi^{*} K_{X_{0}} \cdot \Gamma=\mathscr{D}_{P} \cdot \pi^{*} K_{X_{0}} \cdot L_{3 K}=\pi^{*} K_{K_{0}, F} \cdot{ }_{F} L_{3 K, F}=3 K_{F_{0}}^{2}=3 .
$$

Combining all the inequalities above, we have $K_{S_{0}}^{2} \geq 10$.

Step 4. $\left|K_{S}+\left\lceil\pi^{*} K_{X_{0}}+\mathscr{D}_{P}\right\rceil\right|_{S}$ defines a birational map of $S$.

We consider $\mathscr{M}=\frac{1}{4} \sigma_{S}^{*} K_{S_{0}}+\frac{5}{4} \mathscr{D}_{P, S}$ and $\pi^{*} K_{X_{0}, S}+\mathscr{D}_{P, S} \geq \mathscr{M}$ as before. Now we have

$$
\left(\pi^{*} K_{X_{0}, S}+\mathscr{D}_{P, S}\right)^{2} \geq \mathscr{M}^{2}=\frac{1}{16}\left(K_{S_{0}}^{2}+10 \sigma_{S}^{*} K_{S_{0}} \cdot \mathscr{D}_{P, S}\right) \geq \frac{65}{8}>8 .
$$

Since $\pi^{*} K_{X_{0}} \cdot \Gamma \geq 3$, it follows that $\left|K_{S}+\left\lceil\pi^{*} K_{X_{0}}+\mathscr{D}_{P}\right\rceil\right|_{\Gamma}$ is birational. Thanks to the vanishing of $H^{1}\left(S, K_{S}+\left\lceil\pi^{*} K_{X_{0}}\right\rceil\right)$, it follows that the system $\left|K_{S}+\left\lceil\pi^{*} K_{X_{0}}+\mathscr{D}_{P}\right\rceil\right|_{S}$ separate points on $\Gamma$, that is, general fiber of $S \rightarrow E$. It remains to consider points on different fiber of $S \rightarrow E$. 
We then consider $\mathscr{M} \cdot C$ for a very general curve $C$ on $S$ dominating E.

Suppose that $C^{2} \geq 2$, then $\sigma_{S}^{*} K_{S_{0}} \cdot C \geq 5$ by Hodge Index Theorem. Since $g(C) \geq 2$, then it is clear that $\mathscr{D}_{P, S} \cdot C \geq 2$ for $\left.a\right|_{C}: C \rightarrow E$ has degree $\geq 2$. Hence $\mathscr{M} \cdot C \geq \frac{15}{4}$.

Suppose that $C^{2}=1$, then $\sigma_{S}^{*} K_{S_{0}} \cdot C \geq 4$ by Hodge Index Theorem. Suppose furthermore that $K_{S_{0}} \cdot \sigma_{S}(C)=\sigma_{S}^{*} K_{S_{0}} \cdot C=4$. Since $\sigma_{S}(C)^{2}$ is even and $\sigma_{S}(C)^{2} \geq C^{2}=1$, one sees that $\sigma_{S}(C)^{2} \geq 2$ and leads to a contradiction to Hodge Index Theorem. Therefore $\sigma_{S}^{*} K_{S_{0}} \cdot C \geq 5$ and $\mathscr{M} \cdot C \geq \frac{15}{4}$ in this situation. Suppose that $C^{2}=0$, by Lemma 6.2, one has $\mathscr{M} \cdot C \geq \frac{15}{4}$ clearly. Therefore, for very general curve, we always has

$$
\mathscr{M} \cdot C \geq \frac{15}{4}>\frac{4}{1+\sqrt{1 / 65}} .
$$

We can conclude that $\left|5 K_{X}\right|$ induces a birational map of $X$ by Theorem 2.3 and the last paragraph of the proof of Proposition 6.3.

Proposition 6.6. Assume that $K_{F_{0}}^{2}=3$, then $\mathscr{D}_{P}$ is a general fiber of $a_{X}$. Moreover, $\left|5 K_{X}\right|$ induces a birational map of $X$.

Proof. We proceed with the following setting:

Setting. Since $K_{F_{0}}^{2} \geq 2$, we know that $\left|3 K_{F_{0}}\right|$ is base point free ([BPV, Theorem 5.1]). We recall some notation as in last sections. Let $\Lambda_{3}:=\sigma_{*} L_{3 K, F}$ and $\Delta:=\sigma_{*} \mathscr{D}_{P, F}$. Then $H^{0}\left(F_{0}, 3 K_{F_{0}}\right) \simeq W_{3}^{0} \otimes W_{3}^{+}$, where $W_{3}^{0}$ (resp. $\left.W_{3}^{+}\right)$can be regarded as a subspace of $H^{0}\left(F_{0}, \Lambda_{3}\right)$ (resp. $H^{0}\left(F_{0}, \Delta\right)$ ). Notice that both $\left|W_{3}^{0}\right|$ and $\left|W_{3}^{+}\right|$are free (and so are $\left|\Lambda_{3}\right|$ and $\left.|\Delta|\right)$ by Corollary 4.8,3.

Claim 1. None of the system $\left|\Lambda_{3}\right|$ or $|\Delta|$ defines a pencil.

Suppose first that $\left|W_{3}^{+}\right|$defines a pencil of curve, denoted $G$, of genus $g(G) \geq 2$. Hence $K_{F_{0}} \cdot \Delta=2 g(G)-2$ is even.

Suppose furthermore that $\left|W_{3}^{0}\right|$ defines a pencil of curves as well, then $K_{F_{0}} \cdot \Lambda_{3}$ is even again. However,

$$
9=3 K_{F_{0}}^{2}=K_{F_{0}} \cdot \Lambda_{3}+K_{F_{0}} \cdot \Delta .
$$

Therefore, $\left|W_{3}^{0}\right|$ can not be a pencil. Now consider the situation that $\left|W_{3}^{0}\right|$ defines a generically finite morphism from $h: F_{0} \rightarrow \mathbb{P}^{m}$, where $m=h^{0}\left(W_{3}^{0}\right)-1=\frac{1}{2} P_{3}(F)-1$ and denote by $V$ its image. Clearly, $\operatorname{deg}(V) \geq h^{0}\left(W_{3}^{0}\right)-2$.

Since $\Lambda_{3}^{2} \geq \operatorname{deg}(V) \geq h^{0}\left(W_{3}^{0}\right)-2=\frac{1}{2} P_{3}(F)-2=3$. Suppose that $\operatorname{deg}(V)=3$ then it is a cubic surface in $\mathbb{P}^{4}$, which is not of general type 
and hence $\Lambda_{3}^{2} \geq \operatorname{deg} h \cdot \operatorname{deg} V \geq 6$. In any event, we have that $\Lambda_{3}^{2} \geq 4$. Together with

$27=\Lambda_{3}^{2}+2 \Lambda_{3} \cdot \Delta+\Delta^{2}=\Lambda_{3}^{2}+2\left(\Lambda_{3}+\Delta\right) \cdot \Delta=\Lambda_{3}^{3}+6 K_{F_{0}} \cdot \Delta=\Lambda_{3}^{2}+12(g(G)-1)$,

one sees that $g(G)=2$ and $\Lambda_{3}^{2}=15$. However, by [X1, Théorème 2.2], a surface $F$ of general type with $p_{g}=0$ and a pencil of genus 2 curve should have $K_{F_{0}}^{2} \leq 2$. Hence we get a contradiction. The above argument shows that none of the system $\left|\Lambda_{3}\right|$ or $|\Delta|$ defines a pencil.

Claim 2. Then $\mathscr{D}_{P}$ is a general fiber of $a_{X}$ and $\left|5 K_{X}\right|$ is birational.

Suppose that $K_{F_{0}}^{2}=3$, then $P_{3}(X) \mid P_{3}\left(F_{0}\right)=10$. If $P_{3}(X)=2$ or 5 , then either $\left|\Lambda_{3}\right|$ or $|\Delta|$ is a pencil by Corollary $4.8,1$, which is a contradiction to Claim 1. Since $P_{3}(X)>1$, then we have $P_{3}(X)=10$ and therefore $a_{*} \mathscr{D}_{P}$ is of rank 1 . Therefore, by Corollary 4.8.4, $\mathscr{D}_{P}$ is a general fiber and hence $\left|5 K_{X}\right|$ is birational by Proposition 6.3 .

\subsection{General fiber has $K_{F_{0}}^{2}=5,7,8,9$.}

Note that $X$ is special and by Theorem 5.3 , we will assume furthermore that $P_{2}(X)=1$. Hence we have

$$
\left|2 K_{X}+P\right|=T_{P}+E_{2},
$$

where $T_{P}$ is smooth and nef.

Let $T=T_{P}$ for some general $P$. Considering the short exact sequence

$$
\begin{aligned}
0 \rightarrow K_{X}+\left\lceil 2 \pi^{*} K_{X_{0}}+2 K_{X}+P-E_{2}-T\right\rceil & \rightarrow K_{X}+\left\lceil 2 \pi^{*} K_{X_{0}}+2 K_{X}+P-E_{2}\right\rceil \\
& \rightarrow K_{T}+\left\lceil 2 \pi^{*} K_{X_{0}}\right\rceil_{T} \rightarrow 0 .
\end{aligned}
$$

By Kawamata-Viehweg vanishing theorem, we have the surjectivity of $H^{0}\left(X, K_{X}+\left\lceil 2 \pi^{*} K_{X_{0}}+2 K_{X}+P-E_{2}\right\rceil\right) \rightarrow H^{0}\left(T, K_{T}+\left\lceil 2 \pi^{*} K_{X_{0}}\right\rceil_{T}\right)$.

Lemma 6.7. If $K_{F_{0}}^{2} \geq 5$, then the volume $\operatorname{vol}(T)=T^{3} \geq 10$.

Proof. Since $K_{F_{0}}^{2} \geq 5$, the linear system $\left|2 K_{F_{0}}\right|$ is base point free. Hence $T_{P, F} \sim 2 \sigma^{*} K_{F_{0}}$. It is easy to see that $a_{X *} \mathscr{O}_{X}(T)=a_{X *}\left(\omega_{X}^{2} \otimes P\right)$ is an ample vector bundle $\mathscr{V}$ of degree 1 and of rank $1+K_{F_{0}}^{2}$. By Proposition 2.4, we know that

$$
\operatorname{vol}(T)=T^{3} \geq 3 \frac{T_{F}^{2}}{1+K_{F_{0}}^{2}}=\frac{12 K_{F_{0}}^{2}}{1+K_{F_{0}}^{2}} \geq 10,
$$

when $K_{F_{0}}^{2} \geq 5$.

Lemma 6.8. $P_{3}(X)=\operatorname{dim} W_{3}^{0} \geq 4$. 
Proof. We have seen that $T^{3} \geq 10$. Moreover, $2 \pi^{*} K_{X_{0}} \succeq T$. Hence $\operatorname{vol}(X)=\left(\pi^{*} K_{X_{0}}\right)^{3} \geq \frac{1}{8} T^{3} \geq \frac{5}{4}$. Since $\chi\left(\mathscr{O}_{X}\right)=0$, the Riemann-Roch formula implies that $P_{3}(X) \geq \frac{5}{2} \operatorname{vol}(X) \geq \frac{25}{8}$ and hence $P_{3}(X) \geq 4$.

Lemma 6.9. Suppose that $\chi\left(\mathscr{O}_{X}\right)=0$ and $P_{2}(X)=1$. Then $P_{3}(X) \leq$ 5. Moreover, $P_{3}(X)=5$ if and only if its minimal model $X_{0}$ has at worst Gorenstein singularities.

Proof. Let $X_{0}$ be the minimal model of $X$. By [CC1, 3.5], one has

$$
\sigma\left(X_{0}\right)=10 \chi\left(\mathscr{O}_{X_{0}}\right)+5 \chi_{2}\left(X_{0}\right)-\chi_{3}\left(X_{0}\right)=5-P_{3}(X) \geq 0 .
$$

Moveover, $\sigma\left(X_{0}\right)=0$ if and only if $X_{0}$ has at worst Gorenstein singularities.

By [Theorem 1.1] of [CCZ, $\left|5 K_{X_{0}}\right|$ is birational if $X_{0}$ is minimal with at worst Gorenstein singularities. Moreover, $P_{3}(X) \mid P_{3}(F)$ for special irregular threefolds (cf. Lemma 4.4). Therefore, we conclude that

Corollary 6.10. (1) $\left|5 K_{X}\right|$ is birational unless $\chi\left(\mathscr{O}_{X}\right)=0, P_{2}(X)=$ 1 and $P_{3}(X)=4$.

(2) In the case that $K_{F_{0}}^{2}=7$ or 8 , one has that $\left|5 K_{X}\right|$ is birational.

It remains to consider the following two cases:

Case 1. $K_{F_{0}}^{2}=5, P_{3}(X)=\operatorname{dim} W_{3}^{0}=4$, and $\operatorname{dim} W_{3}^{+}=4$.

Case 2. $K_{F_{0}}^{2}=9, P_{3}(X)=\operatorname{dim} W_{3}^{0}=4$, and $\operatorname{dim} W_{3}^{+}=7$.

Proposition 6.11. In the Case 1 that $K_{F_{0}}^{2}=5,\left|5 K_{X}\right|$ induces a birational map.

Proof. This is a involved case that we need to work harder.

Step 1. The system $\left|5 K_{X}\right|_{T}$ induces a birational map of $T$.

To see this, it suffices to apply Theorem 2.3 to show that $\mid K_{T}+$ $\left\lceil 2 \pi^{*} K_{X_{0}}\right\rceil_{T} \mid$ induces a birational map of $T$. We note that $2 \pi^{*} K_{X_{0}} \succeq T_{P}$ for $P \in \operatorname{Pic}^{0}(E)_{\text {tor }}$. Hence $\left\lceil 2 \pi^{*} K_{X_{0}}\right\rceil_{T} \succeq T_{P, T}$.

We then consider the eventual map of $X$ associated to $\mathscr{O}_{X}(T)$ defined in [J2], which is indeed the relative evaluation morphism of $\mathscr{V}$ :

$$
\varphi: X \rightarrow \mathbb{P}_{E}(\mathscr{V})
$$

We note that $\varphi$ is generically finite, whose restriction to a general fiber $F$ of $a_{X}$ is exactly the bicanonical morphism of $F$. Note that $\varphi^{*} \mathscr{O}_{\mathbb{P}_{E}(\mathscr{V})}(1)=\mathscr{O}_{X}(T)$. Let $C$ be a general curve on $T \subset X$. If $C$ is supported in a fiber $F$ of $a_{X}$, we see that $C \cdot T=2\left(C \cdot \sigma^{*} K_{F_{0}}\right)_{F} \geq 4$.

Suppose that $C \rightarrow E$ is dominant and $C^{2}>0$, then Hodge Index Theorem shows that $C \cdot T \geq 4$. Suppose that $C \rightarrow E$ is dominant and $C^{2}=0$, then Lemma 6.2 asserts that $\operatorname{deg}(C \rightarrow E) \geq 3$. Hence 
$(C \cdot T)_{X} \geq 3$ in any situation. By Theorem 2.3, $\left|5 K_{X}\right|_{T} \geq\left|K_{T}+T_{T}\right|$ is birational.

Step 2. We have $h^{1}\left(\mathscr{O}_{T}\right)=h^{1}\left(\mathscr{O}_{X}\right)=1$ and the restriction of $a_{X}$ on $T$ is $a_{T}$, the Albanese map of $T$.

Since $T$ is a big and nef divisor on $X$, we have $h^{1}\left(\mathscr{O}_{T}\right)=h^{1}\left(\mathscr{O}_{X}\right)=1$. Moreover, since

$$
T_{P, F}+E_{2, F}=\left.\left(2 K_{X}+P\right)\right|_{F}=2 K_{F} \succeq \sigma^{*} 2 K_{F_{0}},
$$

and $E_{2, F}$ is $\sigma$-exceptional for general $F$. One has $T_{P, F}=\sigma^{*} 2 K_{F_{0}}$. This implies in particular that a general fiber $C_{t}$ of $T \rightarrow E$ over $t \in E$ is a connected smooth member of $\left|2 \sigma^{*} K_{F_{0}}\right|$ on $F$. Hence the restriction of $a_{X}$ on $T$ is the Albanese morphism of $T$ and the general fiber is a curve with $g\left(C_{t}\right)=1+3 K_{F_{0}}^{2}$.

Step 3 Also, we have $h^{1}\left(\mathscr{O}_{\mathscr{D}_{P}}\right)=1$. In particular, the Stein factorization of the restriction of $\left.a_{X}\right|_{\mathscr{D}_{P}}$ on $\mathscr{D}_{P}$ is the Albanese map of $a_{\mathscr{D}_{P}}$.

Note that $H^{2}\left(X, \mathscr{O}_{X}\left(-\mathscr{D}_{P}\right)\right)$ is dual to $H^{1}\left(X, K_{X}+\mathscr{D}_{P}\right)$. Since the Iitaka model of $\left(X, \mathscr{D}_{P}\right)$ dominates $E$, we have

$$
h^{1}\left(X, a_{X *} \mathscr{O}_{X}\left(K_{X}+\mathscr{D}_{P}\right)\right)=0
$$

by Kollár's vanishing theorem. Moreover, since $|\Delta|$ is nef and big, a general member of $|\Delta|$ is connected (and hence so is $\left|\mathscr{D}_{P, F}\right|$ ). It follows that $h^{1}\left(F, K_{F}+\mathscr{D}_{P, F}\right)=0$. Since $\mathscr{D}_{P}$ is movable and nef, $R^{1} a_{X *} \mathscr{O}_{X}\left(K_{X}+\mathscr{D}_{P}\right)=0$ by the Kollár's torsion-freeness of $R^{1} a_{X *} \mathscr{O}_{X}\left(K_{X}+\right.$ $\left.\mathscr{D}_{P}\right)$. We then conclude that $1=h^{1}\left(\mathscr{O}_{X}\right)=h^{1}\left(\mathscr{O}_{\mathscr{D}_{P}}\right)$.

Step 4. $\Delta$ and $\Lambda_{3}$ are big and nef.

Suppose on the contrary that $\Delta$ defines a pencil, then $|\Delta|$ defines a morphism $f: F_{0} \rightarrow \mathbb{P}^{1}$ and $\Delta=f^{*} \mathscr{O}_{\mathbb{P}^{1}}(a)$ for some $a \geq 3$. Let $H=f^{*} \mathscr{O}_{\mathbb{P}^{1}}(1)$. From $3 K_{F_{0}}=\Lambda_{3}+a H$, we have

$$
\Lambda_{3} \cdot H=3 K_{F_{0}} \cdot H=6(g(H)-1),
$$

and

$$
45=\left(3 K_{F_{0}}\right)^{2}=\Lambda_{3}^{2}+12 a(g(H)-1) .
$$

By $[\mathrm{X} 1$, Théorème 2.2], $g(H) \geq 3$, hence we get a contradiction. Therefore, $\Delta$ defines a map with 2 -dimensional image. In particular, $\Delta$ is big. The same argument holds for $\Lambda_{3}$ as well.

Step 5. Any dominant maps from $\mathscr{D}_{P}$ to $T$ is birational. 
Assume that there exists a dominant map $\varphi: \mathscr{D}_{P} \rightarrow T$. Since $h^{1}\left(\mathscr{O}_{T}\right)=h^{1}\left(\mathscr{O}_{\mathscr{D}_{P}}\right)$, the Albanese fiber of $a_{\mathscr{D}_{P}}$, denoted $C_{t}^{\prime}$ should also dominates the Albanese fiber $C_{t}$ of $a_{T}$.

Since $\Lambda_{3}$ is a big divisor on $F_{0}$. We claim that $\Lambda_{3}^{2} \geq 4$. To see this, we consider $\varphi_{\Lambda_{3}}: F_{0} \rightarrow Z \subset \mathbb{P}^{3}$. The

$$
\Delta^{2} \geq \operatorname{deg}\left(\varphi_{\Lambda_{3}}\right) \operatorname{deg}(Z) .
$$

If $\operatorname{deg}(Z)=2,3$, then $Z$ is not of general type and hence $\operatorname{deg}\left(\varphi_{\Lambda_{3}}\right) \geq 2$. Therefore, $\Lambda_{3}^{2} \geq 4$.

By Hodge Index Theorem, we have that $K_{F_{0}} \cdot \Lambda_{3} \geq 5$. Recall that

$$
15=3 K_{F_{0}}^{2}=K_{F_{0}} \cdot \Lambda_{3}+K_{F_{0}} \cdot \Delta .
$$

Thus $K_{F_{0}} \cdot \Delta \leq 10$. Then $\Delta^{2} \leq 20$ and equality holds only when $\Delta \equiv 2 K_{F_{0}}$ by Hodge Index Theorem again. Hence

$$
2 g\left(C_{t}^{\prime}\right)-2=K_{F_{0}} \cdot \Delta+\Delta^{2} \leq 30 .
$$

Suppose that $g\left(C_{t}^{\prime}\right)<16$, then we reach a contradiction since $C_{t}^{\prime}$ dominates $C_{t}$ and $g\left(C_{t}\right)=16$. Therefore, $g\left(C_{t}^{\prime}\right)=16$ and $\Delta \equiv 2 K_{F_{0}}$. Moreover, $\mathscr{D}_{P} \rightarrow T$ is birational.

Step 6. The linear system $\left|5 K_{X}\right|$ induces a generically finite map $\varphi$.

After birational modifications, consider $\varphi: X \rightarrow Z \subset \mathbb{P}^{N}$ be the morphism induced by $\left|5 K_{X}\right|$. Assume that $\operatorname{dim} Z \leq 2$. Note that $\left|5 K_{X}\right|_{T}$ induces a birational map from $T$ to its image. Hence $Z$ is birational to $T$ and in particular $q(Z)>0$. Thus $q(Z)=1$. Thus restrict $\varphi$ to a general fiber $F$ of $a_{X}$, we see that the image of $F$ is a general fiber of the Albanese morphism of $Z$ and hence is a curve of genus $g \geq 2$, which is a contradiction to $p_{g}(F)=q(F)=0$.

Step 7. The map $\varphi$ induced by $\left|5 K_{X}\right|$ has degree $\leq 2$.

Let $x \neq y$ be two general points of $X$. Take $P \in \operatorname{Pic}^{0}(X)$ such that $x \in T_{P}$. If $y \in T_{P}$, then by Step $1,\left|5 K_{X}\right|$ separates $x$ and $y$.

If $y \notin T_{P}$, we note that $T_{P}+\mathscr{D}_{-P}+\left|L_{3}\right|$ is a sub-linear system of $\left|5 K_{X}\right|$. Hence if $y \notin \mathscr{D}_{-P}$, then $x$ and $y$ can be separated by $\left|5 K_{X}\right|$.

Suppose now that $y \notin T_{P}, y \in \mathscr{D}_{-P}$. As $\left.\varphi\right|_{T_{P}}$ is birational, $x$ is general and $\varphi(y)=\varphi(x) \in \varphi\left(T_{P}\right)$, we have $\operatorname{dim}\left(\varphi\left(\mathscr{D}_{-P}\right) \cap \varphi\left(T_{P}\right)\right)=2$. Since $\mathscr{D}_{-P}$ is irreducible, it follows that $\mathscr{D}_{-P}$ is an irreducible component of $\varphi^{-1}\left(\varphi\left(T_{P}\right)\right)$ and there exists a dominant map $\mathscr{D}_{-P} \rightarrow T_{P}$. By Step 5 , one sees that $\operatorname{deg}(\varphi) \leq 2$.

Step 8. The map $\varphi$ induced by $\left|5 K_{X}\right|$ is birational. 
Suppose that $\varphi$ has degree 2 , then there exists a birational involution $\sigma$ acting on $X$ such that $\varphi$ is birational to the quotient map $X \rightarrow X / \sigma$. Take a birational model $\rho: \tilde{X} \rightarrow X$ such that we can lift the action $\sigma$ to a biregular involution $\widetilde{\sigma}$ on $\widetilde{X}$. Then $\widetilde{\sigma}_{*} \widetilde{T}_{P}=\widetilde{\mathscr{D}}_{-P}$, where $\widetilde{T}_{P}$ and $\widetilde{D}_{-P}$ are respectively the proper transform of $T_{P}$ and $\mathscr{D}_{-P}$. On the other hand, we always have

$$
\left|2 K_{\widetilde{X}}+P\right|=\widetilde{T}_{P}+E_{2}^{\prime}
$$

and

$$
\left|3 K_{X}-P\right|=\left|\rho^{*} L_{3 K}\right|+\widetilde{\mathscr{D}}_{-P}+E_{3}^{\prime},
$$

where $E_{2}^{\prime}$ and $E_{3}^{\prime}$ are always exceptional divisors from $\widetilde{X}$ to its relative minimal model over $E$. Apply $\widetilde{\sigma}_{*}$ to the first equality, we have $2 K_{\widetilde{X}}+$ $P^{\prime}=\widetilde{\mathscr{D}}_{-P}+\widetilde{\sigma}_{*}\left(E_{2}^{\prime}\right)$, where $P^{\prime}=\widetilde{\sigma}_{*}(P) \in \operatorname{Pic}^{0}(X)$. Hence $K_{\widetilde{X}}-P-P^{\prime}=$ $\rho^{*} L_{3 K}+E_{3}^{\prime}-\widetilde{\sigma}_{*}\left(E_{2}^{\prime}\right)$. Restricted to a general fiber $\widetilde{F}$ of $a_{\widetilde{X}}$, we see that

$$
K_{\widetilde{F}}+\widetilde{\sigma}_{*}\left(E_{2}^{\prime}\right)_{\widetilde{F}}=\left(\rho^{*} L_{3 K}\right)_{F}+E_{3, \widetilde{F}}^{\prime} .
$$

Since $\widetilde{\sigma}_{*}\left(E_{2}^{\prime}\right)_{\widetilde{F}}$ and $E_{3, \widetilde{F}}^{\prime}$ are effective exceptional divisors from $\widetilde{F}$ to its minimal model $F_{0}$. It follows that $h^{0}\left(\widetilde{F}, K_{\widetilde{F}}+\widetilde{\sigma}_{*}\left(E_{2}^{\prime}\right)_{\widetilde{F}}\right)=h^{0}\left(F_{0}, K_{F_{0}}\right)=$ 0 but the right hand side is effective, which is a contradiction.

Proposition 6.12. In the Case 2 that $K_{F_{0}}^{2}=9,\left|5 K_{X}\right|$ induces a birational map.

Proof. We basically use the same approach as in Case 1 with modification on some of the steps. First of all, one can easily sees that Steps 1-3 holds.

Step $4^{\prime}$. Both $\Lambda_{3}$ and $\Delta$ are ample.

When $K_{F_{0}}^{2}=9$, we know that the Néron-Severi group $\mathrm{NS}\left(F_{0}\right) \simeq \mathbb{Z}$. Let $H$ be an ample line bundle on $F_{0}$ such that its class $[H]$ is a generator of $\mathrm{NS}\left(F_{0}\right)$. We know that $h^{0}\left(F_{0}, \Lambda_{3}\right) \geq 4$ and $h^{0}\left(F_{0}, \Delta\right) \geq 4$. Hence both $\Lambda_{3}$ and $\Delta$ are ample.

Step $5^{\prime}$. Any dominant maps from $\mathscr{D}_{P}$ to $T$ is birational.

Let $K_{F_{0}}=k H, \Lambda_{3}=b_{1} H, \Delta=b_{2} H$. It is clear that the only possibilities for $\left(k, H^{2}\right)$ are $(1,9),(3,1)$. A simple computation as in Step 5 shows that if $\mathscr{D}_{P}$ dominates $T$, then it is birational and $\Delta \equiv$ $6 H \equiv 2 K_{F_{0}}, \Lambda_{3} \equiv 3 H \equiv K_{F_{0}}$.

Then the Step 6-8 holds verbatim.

This concludes the proof of the Case 2, and hence the proof of our main theorem that $\left|5 K_{X}\right|$ is birational for any irregular threefold of general type. 
ON QUINT-CANONICAL BIRATIONALITY OF IRREGULAR THREEFOLDS 31

\section{REFERENCES}

[BLNP] M.A. Barja, M. Lahoz, J.C. Naranjo, G. Pareschi, On the bicanonical map of irregular varieties, J. Algebraic Geom. 21 , 2012, 445-471.

[BPS] M.A. Barja, R. Pardini, L. Stoppino, Linear systems on irregular varieties, arXiv: 1606.03290 .

[BPV] W. Barth, C. Peters, A. Van de Ven, Compact complex surfaces, Ergebnisse der Mathematik und ihrer Grenzgebiete 4 (1984), Springer Verlag, BerlinHeidelberg-New York.

[Cat] F. Catanese, Pluricanonical mappings of surfaces with $K^{2}=1,2, q=p_{g}=0$, Algebraic surfaces, 247-266, C.I.M.E. Summer Sch., 76, Springer, Heidelberg, 2010.

[CC1] J. A. Chen, M. Chen, Explicit birational geometry of threefolds of general type, I, Ann. Sci. Ecole Norm. Sup. 43, 2010, 365-394.

[CC2] J. A. Chen, M. Chen, Explicit birational geometry of threefolds of general type, II, J. Differential Geom. 86 (2010), no. 2, 237-271.

[CC3] J. A. Chen, M. Chen, Explicit birational geometry of 3-folds and 4-folds of general type, III, Comp. Math 151 (2015), 1041-1082.

[CCZ] J. A. Chen, M. Chen, D. Q. Zhang The 5-canonical system on 3-folds of general type, J. Reine Angew. Math. 603, 2007, 165-181.

[CH1] J. A. Chen, C. D. Hacon, Characterization of abelian varieties, Invent. math. 143 (2001), 435-447.

[CH2] J. A. Chen, C. D. Hacon, Pluricanonical maps of varieties of maximal Albanese dimension, Math. Ann. 320 (2001), 367-380.

[CH3] J. A. Chen, C. D. Hacon, Pluricanonical systems on irregular 3-folds of general type. Math. Z. 255 (2007), no. 2, 343-355.

[CH4] J. A. Chen, C. D. Hacon, Linear series of irregular varieties, Algebraic geometry in East Asia (Kyoto, 2001), 143-153, World Sci. Publ., River Edge, NJ, 2002.

[C] M. Chen, Canonical stability of 3-folds of general type with $p_{g} \geq 3$, Internat. J. Math. 14 (2003), no. 5, 515-528.

[C18] M. Chen, On minimal 3-folds of general type with maximal pluricanonical section index. Asian J. Math. 22 (2018), no. 2, 257-268.

[D] O. Debarre, Inégalités numériques pour les surfaces de type général, Bull. Soc. Math. France 110 (1982), no. 3, 319-346.

[HM06] C. D. Hacon, J. McKernan. Boundedness of pluricanonical maps of varieties of general type, Invent. Math. 166 (2006), no.1, 1-25.

[J1] Z. Jiang, An effective version of a theorem of Kawamata on the Albanese map, Communications in Contemporary Mathematics Vol. 13, No. 3 (2011) 509-532.

[J2] Z. Jiang,, On Severi type inequalities, arXiv:1901.11207.

[JLT] Z. Jiang, M. Lahoz, L. Tirabassi, On the Iitaka fibration of varieties of maximal Albanese dimension, Int. Math. Res. Not. IMRN 2013, no. 13, 2984-3005.

[JS] Z. Jiang, H. Sun, Cohomological support loci of varieties of Albanese fiber dimension one, Trans. Amer. Math. Soc. 367 (2015), no. 1, 103-119.

[JP] Z. Jiang, G. Pareschi, Cohomological rank functions on abelian varieties, to appear in Annales. E.N.S.

[Ko] J. Kollár, Higher direct image of dualizing sheaves I, Ann. Math. 123 (1986), $11-46$. 
[Laz] R. Lazarsfeld, Lectures on linear series, Complex Algebraic Geometry, IAS/Park City Math. Series 3 (1997), 161-219.

[Lan] A. Langer, Adjoint linear systems on normal log surfaces, Compositio Math. 129 (2001), no. 1, 47-66.

[K] Y. Kawamata, On the extension problem of pluricanonical forms, Algebraic geometry: Hirzebruch 70 (Warsaw, 1998), 193-207, Contemp. Math., 241, Amer. Math. Soc., Providence, RI, 1999.

[Mas] V. Maşek,, Very ampleness of adjoint linear systems on smooth surfaces with boundary, Nagoya Math. J. 153 (1999), 1-29.

$[\mathrm{M}] \mathrm{M}$. Mendes Lopes, The degree of the generators of the canonical ring of surfaces of general type with $p_{g}=0$, Arch. Math. 69 (1997), 435-440.

[MP] M. Mendes Lopes, R. Pardini, The bicanonical map of surfaces with $p_{g}=0$ and $K^{2} \geq 7$, Bull. London Math. Soc. 33 (2001), 1-10.

[PP] G. Pareschi, M. Popa, Regularity on abelian varieties I, J. Amer. Math. Soc. 16 (2003), 285-302.

[PPS] G. Pareschi, M. Popa, Ch. Schnell, Hodge modules on complex tori and generic vanishing for compact Kähler manifolds. Geom. Topol. 21 (2017), no. 4, 2419-2460.

[Taka] S. Takayama. Pluricanonical systems on algebraic varieties of general type. Invent. Math. 165 (2006), no. 3, 551-587.

[Tsuji] H. Tsuji, Pluricanonical systems of projective varieties of general type. I. Osaka J. Math. 43 (2006), no. 4, 967-995.

[X1] G. Xiao, Surfaces fibrées en courbes de genre deux, Lecture Notes in Math. 1137, 1985.

[X2] G. Xiao, Degree of the Bicanonical Map of a Surface of General Type, American Journal of Mathematics, 112, No. 5, 1990, 713-736.

Department of Mathematics, National Central University, ChungLi, TAOYUAN, TAIWAN

E-mail address: jhengjie@math.ncu.edu.tw

Department of Mathematics, National Taiwan University, and Nationnl Center for Theoretical Sciences, Taipei 106, Taiwan

E-mail address: jkchen@ntu.edu.tw

School of Mathematical Sciences, Fudan University, Shanghai 200433, P. R. China

E-mail address: mchen@fudan.edu.cn.

Shanghai Center for Mathematical Sciences, Jiangwan Campus, FuDan University, Shanghai 200438, P. R. China

E-mail address: zhijiang@fudan.edu.cn 